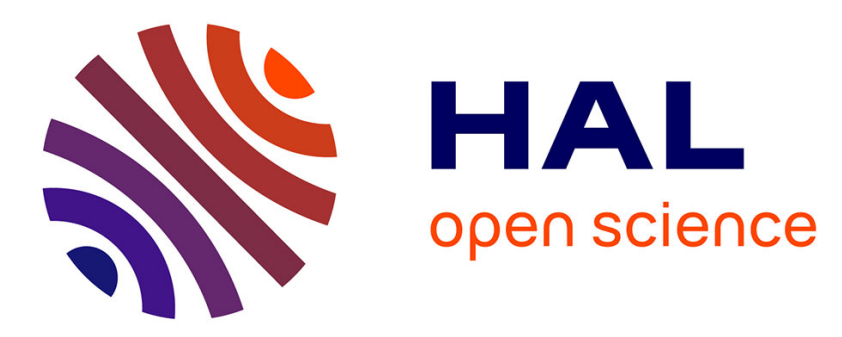

\title{
Dynamics of Political Opinion Formation including Catastrophe Theory
}

\author{
Michael Sonis, Wolfgang Weidlich, Heide Huebner
}

\section{To cite this version:}

Michael Sonis, Wolfgang Weidlich, Heide Huebner. Dynamics of Political Opinion Formation including Catastrophe Theory. Journal of Economic Behavior and Organization, 2008, 67 (1), pp.1. 10.1016/j.jebo.2007.02.002 . hal-00598261

\section{HAL Id: hal-00598261 \\ https://hal.science/hal-00598261}

Submitted on 6 Jun 2011

HAL is a multi-disciplinary open access archive for the deposit and dissemination of scientific research documents, whether they are published or not. The documents may come from teaching and research institutions in France or abroad, or from public or private research centers.
L'archive ouverte pluridisciplinaire HAL, est destinée au dépôt et à la diffusion de documents scientifiques de niveau recherche, publiés ou non, émanant des établissements d'enseignement et de recherche français ou étrangers, des laboratoires publics ou privés. 


\section{Accepted Manuscript}

Title: Dynamics of Political Opinion Formation including Catastrophe Theory

Authors: Michael Sonis, Wolfgang Weidlich, Heide Huebner

PII: $\quad$ S0167-2681(07)00065-0

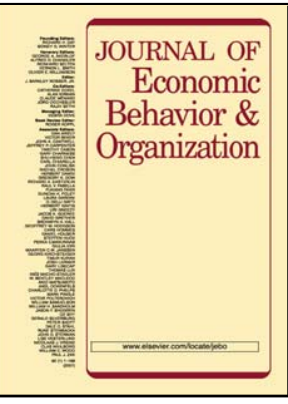

DOI: doi:10.1016/j.jebo.2007.02.002

Reference: $\quad$ JEBO 2091

To appear in: Journal of Economic Behavior \& Organization

Received date: $\quad$ 17-3-2006

Revised date: $\quad 1-11-2006$

Accepted date: $\quad$ 26-2-2007

Please cite this article as: Sonis M., Weidlich W., Huebner H., Dynamics of Political Opinion Formation including Catastrophe Theory, Journal of Economic Behavior and Organization (2007), doi:10.1016/j.jebo.2007.02.002

This is a PDF file of an unedited manuscript that has been accepted for publication. As a service to our customers we are providing this early version of the manuscript. The manuscript will undergo copyediting, typesetting, and review of the resulting proof before it is published in its final form. Please note that during the production process errors may be discovered which could affect the content, and all legal disclaimers that apply to the journal pertain. 


\section{Dynamics of Political Opinion Formation including Catastrophe Theory}

Dedicated to Professor Dr. Michael Sonis on the Occasion of his $70^{\text {th }}$ Birthday

Wolfgang Weidlich ${ }^{1}$ and Heide Huebner ${ }^{2}$

\section{Abstract}

After Nazism and Communism new forms of totalitarianism fostered by religious fundamentalism have arisen at the turn of the present century. In view of this fact we present a parsimonious quantitative model designed to shed some light on the dynamics of the formation of totalitarian systems. The model is constructed according to the principles of "sociodynamics" that belongs to the broad field of NSD (nonlinear dynamic systems theory). It comprises two order parameters and four trend parameters. All of them are socio-politically interpreted. Stationary, stagnant and revolutionary system-phases are exhibited in ten scenarios. The relation to catastrophe theory is discussed.

KEY WORDS: Political Systems; Sociodynamics; NDS (Nonlinear Dynamical Systems), Catastrophe Theory.

\section{General Model Design}

\subsection{Application of Sociodynamic-Principles}

The modeling strategy of sociodynamics is an extension of synergetics (Haken 1977) developed for applications in the social sciences and exhibited in review articles (Weidlich 1991, 2005, 2006) and in complete form in books (Weidlich and Haag 1983, Weidlich 2000). The model has forerunners (Weidlich 1971, 1994). In its present form it includes a full quantitative analysis and sociopolitical interpretation of all key variables and trend parameters as well as the discussion of its relation to another modeling approach, the catastrophe theory.

In short the modeling strategy of sociodynamics consists of three steps:

1. The choice of sociologically interpretable order parameters (i.e. key variables) dominating the dynamics of the system under consideration;

2. The choice of transition rates for the elementary evolution steps of the order parameters. They consist of mobility and attractiveness factors that on their part depend on the key variables and further trend parameters determining the evolution trends;

\footnotetext{
${ }^{1}$ II. Institute of Theoretical Physics, University of Stuttgart, Pfaffenwaldring 57, D-70550 Stuttgart. Tel: +49-711-685-64919, Fax: +49-711-685-64902. Email: Weidlich@theo2.physik.uni-stuttgart.de

${ }^{2}$ University of Applied Sciences Esslingen, Kanalstr. 33, D-73728 Esslingen. Email: Heide.Huebner@gmx.de
} 
3. The derivation of equations of evolution for the key variables. This leads to a nonlinear dynamical system (NDS).

\subsection{Emphasis on Structure and Dynamics}

The model focuses on structure and dynamics of the political system of society by treating political ideologies on a formally equal basis without explicitly giving attention to their ideological content. This implies the strong statement that dynamic phenomena (e.g. the transitions between liberal and totalitarian states of a society) are essentially invariant with regard to the content, however dependent on the degree of open-mindedness respectively dogmatism of involved ideologies.

An important conclusion derived from the model will be that political systems either approach stationary states or traverse sequentially quasi-stationary stagnant states and revolutionary phase-transitions. Ten scenarios will exemplify the different cases. Two of them are comparable to the scenario based on catastrophe theory turning out to be a limiting case. These scenarios have three identical trend parameters whereas the fourth (i.e. the speed parameter) becomes very small to show the relation to catastrophe theory.

\subsection{Catastrophe Theory compared to Sociodynamics}

Let us begin with a short characterization of catastrophe theory: "Catastrophe theory is a special topic within the broader domain of NDS (nonlinear dynamical systems) that pertains to sudden discontinuous changes of events" (Guastello 1995, p. 3). Catastrophe theory (CT) was mainly developed by René Thom (1972). In the following years Zeeman (1977) and Poston and Stewart (1978) introduced and advocated broad interdisciplinary applications of catastrophe theory. Many applications to problems of social science have since appeared, among them articles of T. Farraro (1978), B. R. Flay (1978), J. Bigelow (1982) and S. J. Guastello (1988) treating topics such as discontinuities in psychology, in organizations (such as firms and states) and in economy in terms of catastrophe theory. Good surveys of this work are given in the books of S. J. Guastello (1995) and J. B. Rosser, Jr. (2000).

The explanatory value of CT is given in each of these applications,

- if, for the considered system, relevant dependent variables and control parameters could be identified and measured and

- if, for control parameters that slowly fluctuate around their critical values, the discontinuous jump of the dependent variable could be observed.

The fact that such successfully verifiable parameterizations of catastrophes have been found in physics (Zeeman 1977, Poston and Stewart 1978), biology (Zeeman 1977), psychology (Flay 1978), social sciences (Guastello 1995, Farraro 1978, Bigelow 1982, Guastello 1988) and economics (Rosser 2000) give rise to a further system-theoretical cognitive value of CT: Since comparable discontinuities in those complex systems occur, there evidently exists a repetitive structural similarity between different microscopic, mesoscopic and macroscopic levels of reality. 
Let us now discuss the coincidences and differences between the (independent) approaches of sociodynamics and catastrophe theory, exemplified in detail by our model.

1. Both approaches belong to the domain of nonlinear dynamical systems (NDS).

2. Catastrophe theory is by definition an equilibrium theory. It focuses on the behavior of the dependent variable(s) in the vicinity of discontinuities, where "catastrophic jumps" between different equilibria belonging to the same control parameters can occur.

Sociodynamics instead constructs equations of evolution for the key variables with explicit time dependence in their whole domain of definition. Apart from special cases, this dynamics is more general than gradient dynamics since in general no global potential exists.

3. In both approaches relevant interpretable variables (order parameters) and control parameters (trend parameters) have to be found for each case of application.

In catastrophe theory the dependent variable(s) and control parameters have to be identified in an interpretable manner with parameters of one of $\mathrm{R}$. Thom's catastrophes.

In sociodynamics instead the dynamics of the order parameters is established by introducing their transition rates. These are composed of mobility and utility terms that are functions of the order and control parameters in interpretable form.

4. The present (sociodynamic) model dynamics is more general than the gradient dynamics. However among the dynamical modes described by the model (see section 3.6 and 3.7) there are embedded two scenarios 7 and 8 leading in the limit case to the cusp catastrophe of CT. In theses cases one order parameter, namely $x$, becomes extremely slow. $x$ and $\kappa$ now serve as control parameters, and there is only one remaining order parameter $y(\tau)$, whose dynamics is now derivable from an even globally constructible potential. The dynamics of these cases comprises the cusp-catastrophe and consists of a completely traversed hysteresis loop with stagnant and revolutionary phases.

\section{The Mathematical Form of the Model}

\subsection{Political Opinions}

By definition, a political opinion is a more or less consistent view or perspective under which a social system is seen. In our stylized, parsimonious treatment we assume two main opinions "+" and "-" only, which appear formally symmetric and without preceding preferences for one of them. In a liberal system they may be identified with the "conservative" and the "progressive" opinion of a two-party system, and in a totalitarian system with the "official state ideology" and the suppressed "dissident opinion". In the liberal system there exist overlaps, compromises and exchange of ideas between the political philosophies behind the "opinions", whereas the ideologies of the totalitarian system are degenerate philoso- 
phies (Weltanschauungen), being dogmatic, fundamentalistic, closed and without compromise. We do not explicitly consider the content of the different types of opinions, but their influence on the structure and the political dynamics of the social system.

\section{$2.2 \quad$ Key Variables}

We now introduce key variables that are considered as the "order parameters" of the political system, dominating its structure and dynamics. We need collective variables for the macro level, being extensive (i. e. proportional to the size of the system), and variables referring to each individual for the micro level, being intense variables independent of the system size. Their interaction will represent the bottom-up and top-down relation between micro and macro level.

The following variables are chosen as they turn out to be the relevant ones dominating the dynamics of societies on the verge of a transition between a liberal and a totalitarian state. The relevance of just these variables is confirmed by insiders who have lived in totalitarian systems, among them Victor Klemperer (1999) and Wolfgang Leonhard (1979).

\subsubsection{The Collective Variables}

We introduce a society with $2 N$ members:

$$
\left.\begin{array}{l}
n_{+}=\text {Number of people exhibiting publicly their opinion "+" } \\
n_{-}=\text {Number of people exhibiting publicly their opinion "-" } \\
n=\frac{1}{2}\left(n_{+}-n_{-}\right) \quad=\text { Majority variable. }
\end{array}\right\}
$$

We assume that no "indifferent" people exist. Then the trivial relations hold:

$$
\begin{array}{ll}
n_{+}+n_{-}=2 N & n_{+}=N+n \\
n_{+}-n_{-}=2 n & n_{-}=N-n \\
-N \leq n \leq+N . &
\end{array}
$$

\subsubsection{The Individual's Variables}

To each individual we assign an inner inclination variable $\vartheta$ describing his inner estimation and valuation of his own publicly exhibited opinion, where $\vartheta>0$ means positive estimation and $\vartheta<0$ negative estimation of his own publicly exhibited opinion. (We anticipate that, particularly in totalitarian systems, the inner inclination of an individual needs not necessarily be in affirmative relation to its publicly exhibited opinion). Thus we introduce

$\left.\begin{array}{l}\vartheta_{+} \text {inner inclination of individuals with exhibited opinion " + " } \\ \vartheta_{-} \quad \text { inner inclination of individuals with exhibited opinion " }-" .\end{array}\right\}$ 
For simplicity we assume the same value $\vartheta_{+}$and $\vartheta_{-}$for all individuals in opinions "+" and "-" respectively.

Further plausible assumptions are now introduced for $\vartheta_{+}$and $\vartheta_{-}$:

- We assume for $\vartheta_{+}$and $\vartheta_{-}$positive and negative integer values only.

- Political psychology indicates that there is a positive as well as negative limit of the inner inclination or disinclination to the own exhibited opinion. This is expressed by

$$
\begin{aligned}
& -\Theta \leq \vartheta_{+} \leq+\Theta \\
& -\Theta \leq \vartheta_{-} \leq+\Theta .
\end{aligned}
$$

- There exists a general trend for the inner inclinations of all individuals. If individuals with opinion "+" (which may be enforced by opinion pressure) are dissatisfied with their own exhibited opinion, they have the inner inclination $\vartheta_{+}<0$. In this case the individuals with the "dissident" opinion "-" will be satisfied with their exhibited opinion (i. e. they have $\vartheta_{-}>0$ ). This antagonistic relation between "+" and "-" can be generalized so that we generally assume

$$
\vartheta_{-}=-\vartheta_{+} \quad \text { or } \quad \vartheta_{+}+\vartheta_{-}=0,
$$

by introducing now one variable of inner inclination only

$$
\vartheta=\vartheta_{+}=-\vartheta_{-} .
$$

The relevant political variables of the system now constitute the configuration

$$
\mathcal{W}=\left\{n_{+}, n_{-} ; \vartheta_{+}, \vartheta_{-}\right\}
$$

Taking into account (2.2) and (2.6) this reduces to

$$
\mathcal{W}=\{n ; \vartheta\}
$$

\subsection{Transitions and Transition Probabilities}

The whole dynamics of the system evolves out of a sequence of elementary transitions, each starting from a given initial configuration $\mathcal{W}_{i}$ and ending in one of neighboring configurations $\mathcal{W}_{n_{\uparrow}}, \mathcal{W}_{n_{\downarrow}}, \mathcal{W}_{\vartheta \uparrow}, \mathcal{W}_{\vartheta \downarrow}$. Starting from $\mathcal{W}_{i}$ equal to (2.7), these transitions are

$$
\begin{aligned}
& \mathcal{W} \rightarrow \mathcal{W}_{n \uparrow}=\left\{n_{+}+1, n_{-}-1 ; \vartheta_{+}, \vartheta_{-}\right\} \hat{=}\{n+1, \vartheta\} \\
& \mathcal{W} \rightarrow \mathcal{W}_{n \downarrow}=\left\{n_{+}-1, n_{-}+1 ; \vartheta_{+}, \vartheta_{-}\right\} \hat{=}\{n-1, \vartheta\} \\
& \mathcal{W} \rightarrow \mathcal{W}_{\vartheta \uparrow}=\left\{n_{+}, n_{-} ; \vartheta_{+}+1, \vartheta_{-}-1\right\} \hat{=}\{n, \vartheta+1\} \\
& \mathcal{W} \rightarrow \mathcal{W}_{\vartheta \downarrow}=\left\{n_{+}, n_{-} ; \vartheta_{+}-1, \vartheta_{-}+1\right\} \hat{=}\{n, \vartheta-1\} .
\end{aligned}
$$

They correspond to 
$\mathcal{W} \rightarrow \mathcal{W}_{n \uparrow}$ : The transition of one individual from opinion " -" to opinion " +";

$\mathcal{W} \rightarrow \mathcal{W}_{n \downarrow}$ : The transition of one individual from opinion " +" to opinion " -";

$\mathcal{W} \rightarrow \mathcal{W}_{\vartheta \uparrow}: \quad$ The transition of each individual from inner inclination $\vartheta$ to inner inclination $(\vartheta+1)$;

$\mathcal{W} \rightarrow \mathcal{W}_{\vartheta \downarrow}: \quad$ The transition of each individual from inner inclination $\vartheta$ to inner inclination $(\vartheta-1)$.

The transitions (2.8) are engendered by transition probabilities (per unit of time) of individuals from their initial position to their final position namely by
(a) $p_{n \uparrow}$
(b) $p_{n \downarrow}$
(c) $p_{\vartheta \uparrow}$
(d) $p_{\vartheta \downarrow}$

It has been proven mathematically (Weidlich 2006) that quite generally individual transition probabilities from an initial state " $i$ " to a final state " $f$ " of the individual have the form

$$
p_{i \rightarrow f}=\mu\left(\mathcal{W}_{i}, \mathcal{W}_{f}\right) \exp \left(u_{f}\left(\mathcal{W}_{f}\right)-u_{i}\left(\mathcal{W}_{i}\right)\right)
$$

with $\mu\left(\mathcal{W}_{i}, \mathcal{W}_{f}\right)=\mu\left(\mathcal{W}_{f}, \mathcal{W}_{i}\right)$

This mathematical form shows that the transition probabilities $p_{i \rightarrow f}$ consist of two factors:

- $\mu\left(\mathcal{W}_{i}, \mathcal{W}_{f}\right)$ the mobility factor, and

- $\exp \left(u_{f}\left(\mathcal{W}_{f}\right)-u_{i}\left(\mathcal{W}_{i}\right)\right)$ the attractiveness factor,

which favors the transition $i \rightarrow f$ if $u_{f}\left(\mathcal{W}_{f}\right)>u_{i}\left(\mathcal{W}_{i}\right)$

and disfavors this transition if $u_{f}\left(\mathcal{W}_{f}\right)<u_{i}\left(\mathcal{W}_{i}\right)$.

Therefore the functions $u_{i}\left(\mathcal{W}_{i}\right)$ and $u_{f}\left(\mathcal{W}_{f}\right)$ of the initial (final) state of the individual and the initial (final) configuration, respectively, must be interpreted as measures of the comparative attractiveness or "utility" of the initial and final state for this individual. Therefore they are denoted as dynamical utility functions.

We denote the utility function for the transition probabilities $p_{n \uparrow}$ and $p_{n \downarrow}$ by $u$ and those for $p_{\vartheta \uparrow}$ and $p_{\vartheta \downarrow}$ by $s$. The mobility factors for the transition probabilities $p_{n \uparrow}$ and $p_{n \downarrow}$ are $\hat{\nu}$ and those for $p_{\vartheta \uparrow}$ and $p_{\vartheta \downarrow}$ are $\tilde{\mu}$.

$$
\begin{aligned}
& p_{n \uparrow}(n, \vartheta)=\hat{\nu} \cdot \exp \left(u_{+}\left(n_{+}+1, \vartheta_{+}\right)-u_{-}\left(n_{-}, \vartheta_{-}\right)\right) \\
& p_{n \downarrow}(n, \vartheta)=\hat{\nu} \cdot \exp \left(u_{-}\left(n_{-}+1, \vartheta_{-}\right)-u_{+}\left(n_{+}, \vartheta_{+}\right)\right) \\
& p_{\vartheta \uparrow}(n, \vartheta)=\tilde{\mu} \cdot(\Theta-\vartheta) \cdot \exp (s(n, \vartheta+1)-s(n, \vartheta)) \\
& p_{\vartheta \downarrow}(n, \vartheta)=\tilde{\mu} \cdot(\Theta+\vartheta) \cdot \exp (s(n, \vartheta-1)-s(n, \vartheta)) .
\end{aligned}
$$

The additional limitation factors $(\Theta-\vartheta)$ and $(\Theta+\vartheta)$ in equations $(2.11)(\mathrm{c})$ and $(\mathrm{d})$ have been inserted according to the requirement (2.4) so $\vartheta$ never leaves the domain (2.4). The factors $\hat{\nu}$ and $\tilde{\mu}$ introduced in (2.11) belong to the trend parameters discussed in section 2.5 . 


\subsection{The Dynamical Utility Functions}

At first we mention that our concept of dynamical utility functions should not be confused with the utility functions used in neoclassical economics. The latter are static functions to be maximized in order to describe the equilibrium state of the economic system. Our utility functions instead appear in a dynamical context. Their maximization is not intended. However it can (but needs not) happen that the system approaches a stationary state.

The dynamical utility functions, which are measures of the attractiveness of the configuration $\mathcal{W}=\left\{n_{+}, n_{-} ; \vartheta_{+}, \vartheta_{-}\right\} \hat{=} \mathcal{W}=\{n ; \vartheta\}$ to individuals of opinion "+" or "-", respectively, must now be explicitly chosen in order to complete the model. The choice involves the account of political psychology of individuals under different global situations of the society, such as a liberal, a polarized or a totalitarian situation.

We make the following (most simple) choice:

$$
\begin{aligned}
u_{+}\left(n_{+}, \vartheta_{+}\right) & =\frac{1}{2}\left(\tilde{\kappa} n_{+}+\tilde{\gamma} \vartheta_{+}\right) \\
u_{-}\left(n_{-}, \vartheta_{-}\right) & =\frac{1}{2}\left(\tilde{\kappa} n_{-}+\tilde{\gamma} \vartheta_{-}\right) \\
s(n, \vartheta) & =s_{+}\left(n_{+}, \vartheta_{+}\right)=s_{-}\left(n_{-}, \vartheta_{-}\right)=\tilde{\beta} n \vartheta .
\end{aligned}
$$

Inserting into the transition probabilities (2.11) we get the final form:

$$
\begin{aligned}
p_{n \uparrow}(n, \vartheta) & =\tilde{\nu} \exp (\tilde{\kappa} n+\tilde{\gamma} \vartheta) \\
p_{n \downarrow}(n, \vartheta) & =\tilde{\nu} \exp (-(\tilde{\kappa} n+\tilde{\gamma} \vartheta)) \\
p_{\vartheta \uparrow}(n, \vartheta) & =\tilde{\mu} \cdot(\Theta-\vartheta) \cdot \exp (\tilde{\beta} n) \\
p_{\vartheta \downarrow}(n, \vartheta) & =\tilde{\mu} \cdot(\Theta+\vartheta) \cdot \exp (-\tilde{\beta} n) \\
\text { with } \quad \tilde{\nu} & =\hat{\nu} \exp (\tilde{\kappa} / 2) .
\end{aligned}
$$

where the trend parameters (i. e. control parameters) $\tilde{\nu}, \tilde{\mu}, \tilde{\kappa}, \tilde{\gamma}, \tilde{\beta}$ may assume values in the following domains:

$$
\begin{array}{ll}
0<\tilde{\nu}<\infty & 0<\tilde{\mu}<\infty \\
0<\tilde{\kappa}<\infty & 0<\tilde{\gamma}<\infty \quad-\infty<\tilde{\beta}<+\infty .
\end{array}
$$

\subsection{The Interpretation of Trend Parameters}

The form of the utility functions (2.12) and also of the trend parameters $\tilde{\kappa}, \tilde{\gamma}, \tilde{\beta}, \tilde{\nu}, \tilde{\mu}$ has a straight forward interpretation.

\subsubsection{Opinion Pressure Parameter $\tilde{\kappa}$}

The utility function $u_{+}\left(n_{+}, \vartheta_{+}\right)$of opinion "+" for individuals having this opinion increases due to its first term $\frac{1}{2} \tilde{\kappa} n_{+}$with increasing values of $\tilde{\kappa}$ and an increasing 
number of people $n_{+}$exhibiting publicly the opinion "+". The corresponding holds for people with opinion "-".

The equations (2.13) (a) and (b) show that a large value of $\tilde{\kappa}$ and a large majority $n$ favors evidently the individual's opinion transition probability $p_{n \uparrow}(-\rightarrow+)$ and disfavors the inverse transition probability $p_{n \downarrow}(+\rightarrow-)$. That means the collective opinion distribution represented by the majority variable $n$ plays a growing role for the individual opinion choice by producing a pressure to align to the majority, the higher the numerical value of $\tilde{\kappa}$ is.

Therefore we denote the trend parameter $\tilde{\kappa}$ as the "opinion pressure parameter".

\subsubsection{Inclination Influence Parameter $\tilde{\gamma}$}

The utility $u_{+}\left(n_{+}, \vartheta_{+}\right)$contains another additive term $\frac{1}{2} \tilde{\gamma} \vartheta_{+}$, where $\tilde{\gamma}>0$.

For $\vartheta_{+}=\vartheta>0$ the inner inclination of the individual is affirmative that means it supports its publicly pronounced opinion " + ". This affirmation enhances the utility $u_{+}$and also the transition probability $p_{n \uparrow}$, whereas the inverse transition probability $p_{n \downarrow}$ is diminished. On the other hand for $\vartheta_{+}=\vartheta<0$ the inner inclination is in opposition to the publicly pronounced opinion " + ". The term $\frac{1}{2} \tilde{\gamma} \vartheta_{+}$ reduces the utility function $u_{+}$and also the transition probability $p_{n \uparrow}$, whereas the inverse transition probability $p_{n \downarrow}$ now increases. In any case, growing values of the trend parameter $\tilde{\gamma}$ lead to growing influence of the inner inclination on the choice of the publicly exhibited opinion of the individual.

Therefore we denote $\tilde{\gamma}$ as the "inclination influence parameter".

\subsubsection{Approval and Disapproval Trend Parameter $\tilde{\beta}$}

The trend parameter $\tilde{\beta}$ is most easily discussed in terms of the transition probabilities (2.13) (c) and (d). $\tilde{\beta}$ can be positive or negative. Let it at first be $\tilde{\beta}>0$, then

- if the majority $n$ is positive the transition to positive $\vartheta$ prevails, and

- for negative $n$ the transition to negative $\vartheta$ is favored.

In any case for $\tilde{\beta}>0$ the inner inclination $\vartheta$ is drawn to affirmation of the opinion of the majority. On the other hand for $\tilde{\beta}<0$ the inner inclination $\vartheta$ is drawn into opposition (inner disinclination) to the majority opinion.

Therefore we denote $\tilde{\beta}>0$ as the approval trend, and $\tilde{\beta}<0$ the disapproval trend.

\subsubsection{The speed parameters $\tilde{\nu}$ and $\tilde{\mu}$}

The transition probabilities $p_{n \uparrow}$ and $p_{n \downarrow}$ are proportional to the parameter $\tilde{\nu}$, and those for the inner inclinations $p_{\vartheta \uparrow}$ and $p_{\vartheta \downarrow}$ are proportional to $\tilde{\mu}$. These two trend parameters determine essentially the speed under which the switches between the externally exhibited opinions "+", "-", and the changes of the inner inclination $\vartheta$ take place. Accordingly they are denoted as speed parameters. 


\subsubsection{Composition of the Trend Parameters}

As already mentioned many factors merge into the values of the trend parameters, where some are caused by the regime (i. e. the ruling political system) and some by the individual members of the society. We indicate a qualitative factor analysis for $\tilde{\kappa}, \tilde{\gamma}$ and $\tilde{\beta}$ in the case of a totalitarian regime (that means in the case of large $\tilde{\kappa}$, weak $\tilde{\gamma}$ and negative $\tilde{\beta}$ ).

The opinion pressure $\tilde{\kappa}$ is preferentially regime-induced by propaganda, granting privileges to supporters, and discrimination and exertion of scare tactics against dissidents, and partially induced by individuals by opportunism, protection of family and friends, cowardice, authoritarianism (i. e. obedience to authorities irrespective of their legitimacy). The regime-induced factors are dominant because the behavior of people is mainly a consequence of measures of the regime.

The inclination influence $\tilde{\gamma}$ is partially regime-induced by its attempt to diminish $\tilde{\gamma}$ (i. e. to suppress political influence of people) but preferentially induced by individuals trying to enhance $\tilde{\gamma}$ by becoming aware of the futility of "inner emigration" and by becoming increasingly fierce in the wish to topple the existing regime.

The disapproval trend $\tilde{\beta}<0$ is regime-induced by the arousal of dissatisfaction with the bad material and suppressive political situation leading to inner opposition against injustice, and induced by individuals by independent judgement of the situation and by moral doubts due to increasing awareness of the discrepancy between propaganda and reality.

\subsection{Equations of Evolution}

The equations of evolution for the key variables $\left\{n_{+}, n_{-}, \vartheta_{+}, \vartheta_{-}\right\}$describe the time dependence of $\{n, \vartheta\}$. They depend on the key variables themselves and their transition rates. Since $n_{-}$individuals can make the opinion transition $(-\rightarrow+)$ via $p_{n \uparrow}$ and $n_{+}$individuals the transition $(+\rightarrow-)$ via $p_{n \downarrow}$ we obtain for the mean rate of change of the numbers $n_{+}(t)$ and $n_{-}(t)$ :

$$
\begin{aligned}
& \frac{d n_{+}}{d t}=n_{-} p_{n \uparrow}(n, \vartheta)-n_{+} p_{n \downarrow}(n, \vartheta) \\
& \frac{d n_{-}}{d t}=-n_{-} p_{n \uparrow}(n, \vartheta)+n_{+} p_{n \downarrow}(n, \vartheta)
\end{aligned}
$$

which can be reduced by inserting (2.2) to one equation

$$
\begin{aligned}
\frac{d n}{d t} & =n_{-} p_{n \uparrow}(n, \vartheta)-n_{+} p_{n \downarrow}(n, \vartheta) \\
& =(N-n) p_{n \uparrow}(n, \vartheta)-(N+n) p_{n \downarrow}(n, \vartheta)
\end{aligned}
$$

or explicitly by making use of (2.13) (a) and (b) to

$$
\frac{d n}{d t}=\tilde{\nu}\{(N-n) \exp (\tilde{\kappa} n+\tilde{\gamma} \vartheta)-(N+n) \exp (-(\tilde{\kappa} n+\tilde{\gamma} \vartheta))\}
$$


Furthermore we obtain for the mean rate of change of $\vartheta$

$$
\frac{d \vartheta_{+}}{d t}=-\frac{d \vartheta_{-}}{d t}=\frac{d \vartheta}{d t}=p_{\vartheta \uparrow}(n, \vartheta)-p_{\vartheta \downarrow}(n, \vartheta),
$$

which leads by inserting (2.13) (c) and (d) to

$$
\frac{d \vartheta}{d t}=\tilde{\mu}\{(\Theta-\vartheta) \exp (\tilde{\beta} n)-(\Theta+\vartheta) \exp (-\tilde{\beta} n)\} .
$$

It is now convenient to go to scaled variables,

$$
y=\frac{n}{N} \quad x=\frac{\vartheta}{\Theta},
$$

which vary, according to (2.2) and (2.4) in the domains

$$
-1 \leq y \leq+1 \quad-1 \leq x \leq+1
$$

We also introduce scaled trend parameters

$$
\kappa=N \tilde{\kappa} \quad \gamma=\Theta \tilde{\gamma} \quad \beta=N \tilde{\beta}
$$

defined according to (2.14) in the domains

$$
0 \leq \kappa<\infty \quad 0 \leq \gamma<\infty \quad-\infty<\beta<+\infty .
$$

Furthermore we introduce a dimensionless time variable

$$
\tau=(\tilde{\mu}+\tilde{\nu}) t
$$

as well as dimensionless speed parameters

$$
\nu=\frac{\tilde{\nu}}{\tilde{\nu}+\tilde{\mu}} \quad \mu=\frac{\tilde{\mu}}{\tilde{\nu}+\tilde{\mu}} \quad \text { with } \quad \nu+\mu=1 \quad \text { and } \quad \nu, \mu>0 .
$$

We mention that the interval $\Delta \tau=1$ in the dimensionless time corresponds to the interval $\Delta t=1 /(\tilde{\nu}+\tilde{\mu})$ in the real time $t$.

Then the two relevant equations of evolution (2.17) and (2.19) assume the form

$$
\begin{aligned}
& \frac{d y}{d \tau}=\nu\{(1-y) \exp (\kappa y+\gamma x)-(1+y) \exp (-(\kappa y+\gamma x))\} \quad \text { and } \\
& \frac{d x}{d \tau}=\mu\{(1-x) \exp (\beta y)-(1+x) \exp (-\beta y)\} .
\end{aligned}
$$

Equations (2.26) and (2.27) are a system of coupled nonlinear differential equations. Their structure and content will be considered analytically and numerically in the following section. 


\section{Analysis of the Model}

\subsection{The Procedure}

Although the model is as parsimonious as possible, it nevertheless comprises a manifold of scenarios corresponding to the choice of different sets of the trend parameters $\kappa, \gamma, \beta, \mu, \nu$. The different scenarios not only differ in quantitative details but also in global structure and dynamics. Therefore it is appropriate to proceed systematically in exhausting the content of the model:

- In section 3.2 we show that the variables $y(\tau)$ and $x(\tau)$ cannot leave their domain of definition (2.21) during their evolution. Furthermore we point out the asymmetric role of the variables $x(\tau)$ and $y(\tau)$ in their equations of evolution.

- In section 3.3 we implement a conventional linear stability analysis for the "neutral state" $(y, x)=(0,0)$, which turns out to be a stationary state for all choices of the trend parameters. The question of stability or instability of the neutral state is important for the global dynamics of the system.

- In section 3.4 the equilibrium lines are introduced, where one of the variables $y(\tau)$ or $x(\tau)$ come to rest. Stationary points, where both variables $y(\tau)$ and $x(\tau)$ come to rest, are the points of intersection of the equilibrium lines.

- In section 3.5 the so-called cusp-curve is defined, which will prove to be of special importance in the context of catastrophe theory (section 4).

- In section 3.6 the flux lines are introduced in the plane of variables $(x, y)$. Their differential equation is derived from the fundamental equations of evolution after elimination of time. Specific sets of parameters $\{\kappa, \gamma, \beta, \mu, \nu\}$ are selected in order to comprise all structurally different scenarios of possible global dynamics of the model-system.

- Finally in section 3.7 the flux lines together with the socio-political interpretation of the scenarios selected in section 3.6 are presented on the basis of the trend parameter sets pertaining to them.

\subsection{The Domain of Definition and the Role of the Key- Variables}

According to their original definition (2.1), (2.4), (2.6) and after the transition to scaled variables $(2.20)$, the variables $x(\tau)$ and $y(\tau)$ are meaningful only in their domain of definition (see (2.21)):

$$
-1 \leq x \leq+1 ; \quad-1 \leq y \leq+1 .
$$

The equations of evolution (2.26) and (2.27) are repeated here for convenience:

$$
\begin{aligned}
& \frac{d x}{d \tau}=\mu\{(1-x) \exp (\beta y)-(1+x) \exp (-\beta y)\} \quad \text { and } \\
& \frac{d y}{d \tau}=\nu\{(1-y) \exp (\kappa y+\gamma x)-(1+y) \exp (-(\kappa y+\gamma x))\}
\end{aligned}
$$


They must be compatible with (3.1) in the following sense. Starting from initial values within the domain of definition (3.1) the variables $x(\tau)$ and $y(\tau)$ can never leave their domain during their evolution. This condition is fulfilled as according to $(3.2)$ and $(3.3)$

$$
\begin{array}{ll}
\left.\frac{d x}{d \tau}\right|_{x=1}=-2 \mu \exp (-\beta y)<0 ; & \left.\frac{d x}{d \tau}\right|_{x=-1}=+2 \mu \exp (\beta y)>0 \\
\left.\frac{d y}{d \tau}\right|_{y=1}=-2 \nu \exp (-(\kappa y+\gamma x))<0 ; & \left.\frac{d y}{d \tau}\right|_{y=-1}=+2 \nu \exp (\kappa y+\gamma x)>0 .
\end{array}
$$

From the general structure of equations (3.2) and (3.3) we can now infer that the roles of the "individual's variable" $x(\tau)$ and the collective "majority variable" $y(\tau)$ are not symmetrical:

- The rate of $x(\tau)$ is only influenced by the majority variable $y(\tau)$ but not (apart from the limitation factors $(1 \pm x)$ ) by itself. The reason is that inner inclinations of individuals do not have a direct influence on the inner inclinations of other individuals.

- In contrast to that, the rate of $y(\tau)$ is influenced by the inner inclination of people $x(\tau)$ and also by $y(\tau)$ itself. The reason is that the collective reacts on itself by exerting a public self interaction via opinion pressure. This leads to self organization effects.

Anticipating the results we shall see in detail that for small $\kappa$ and large $\gamma$ the majority variable $y(\tau)$ follows the inner inclination $x(\tau)$, so $x(\tau)$ can be seen to be the "causative" variable in this case. However in the very different case of large $\kappa$ and small $\gamma$ the variable $y(\tau)$ develops its own self-stabilizing dynamics. In this case $x(\tau)$ is practically without influence on $y(\tau)$ apart from "revolutionary events".

\subsection{Stability Analysis of the "Neutral State"}

We begin the analysis of the equations of evolution (3.2) and (3.3) with the "neutral state" $(x, y)=(0,0)$ of balanced inner inclinations and collectively exhibited opinions.

It is easily seen that $(0,0)$ is in all cases a stationary state because

$$
\frac{d x}{d \tau}=0 \quad \text { and } \quad \frac{d y}{d \tau}=0 \quad \text { for } \quad(x, y)=(0,0)
$$

holds for all values of the parameters $\kappa, \gamma, \beta, \mu, \nu$.

In order to decide which combinations of trend parameters lead to a stable or unstable neutral state, we make a standard linear stability analysis. In the vicinity of $(x, y)=(0,0)$ that means for $|x| \ll 1$ and $|y| \ll 1$ we can linearize the equations of evolution (3.2) and (3.3) and obtain

$$
\frac{d x}{d \tau}=-2 \mu x+2 \mu \beta y \quad \text { and }
$$




$$
\frac{d y}{d \tau}=2 \nu \gamma x+2 \nu(\kappa-1) y
$$

The linear differential equations (3.7) and (3.8) can be solved by

$$
x=x_{0} e^{\lambda \tau} \quad \text { and } \quad y=y_{0} e^{\lambda \tau}
$$

where the possible values of $\lambda$ have to fulfil the eigenvalue equation

$$
\left|\begin{array}{cc}
-(2 \mu+\lambda) & 2 \mu \beta \\
2 \nu \gamma & (2 \nu \rho-\lambda)
\end{array}\right|=\lambda^{2}-2(\nu \rho-\mu) \lambda-4 \nu \mu(\gamma \beta+\rho)=0
$$

with $\rho=\kappa-1$. The quadratic equation (3.10) for the eigenvalues $\lambda$ has two solutions, the roots $\lambda_{+}$and $\lambda_{-}$, where

$$
\begin{aligned}
& \lambda_{ \pm}=\nu\left[(\rho-\hat{\mu}) \pm \sqrt{(\rho-\hat{\mu})^{2}+4 \hat{\mu}(\gamma \beta+\rho)}\right] \\
& \text { or } \quad \lambda_{ \pm}=\nu\left[\varphi \pm \sqrt{\varphi^{2}+\psi}\right] \\
& \text { with } \quad \rho=\kappa-1 ; \quad \hat{\mu}=\frac{\mu}{\nu} ; \quad \varphi=\rho-\hat{\mu} ; \quad \psi=4 \hat{\mu}(\gamma \beta+\rho) .
\end{aligned}
$$

From the general solution of (3.7) and (3.8)

$$
x(\tau)=x_{0+} e^{\lambda_{+} \tau}+x_{0-} e^{\lambda_{-} \tau} ; \quad y(\tau)=y_{0+} e^{\lambda_{+} \tau}+y_{0-} e^{\lambda_{-} \tau},
$$

we have to distinguish the following cases for the stability of the neutral state:

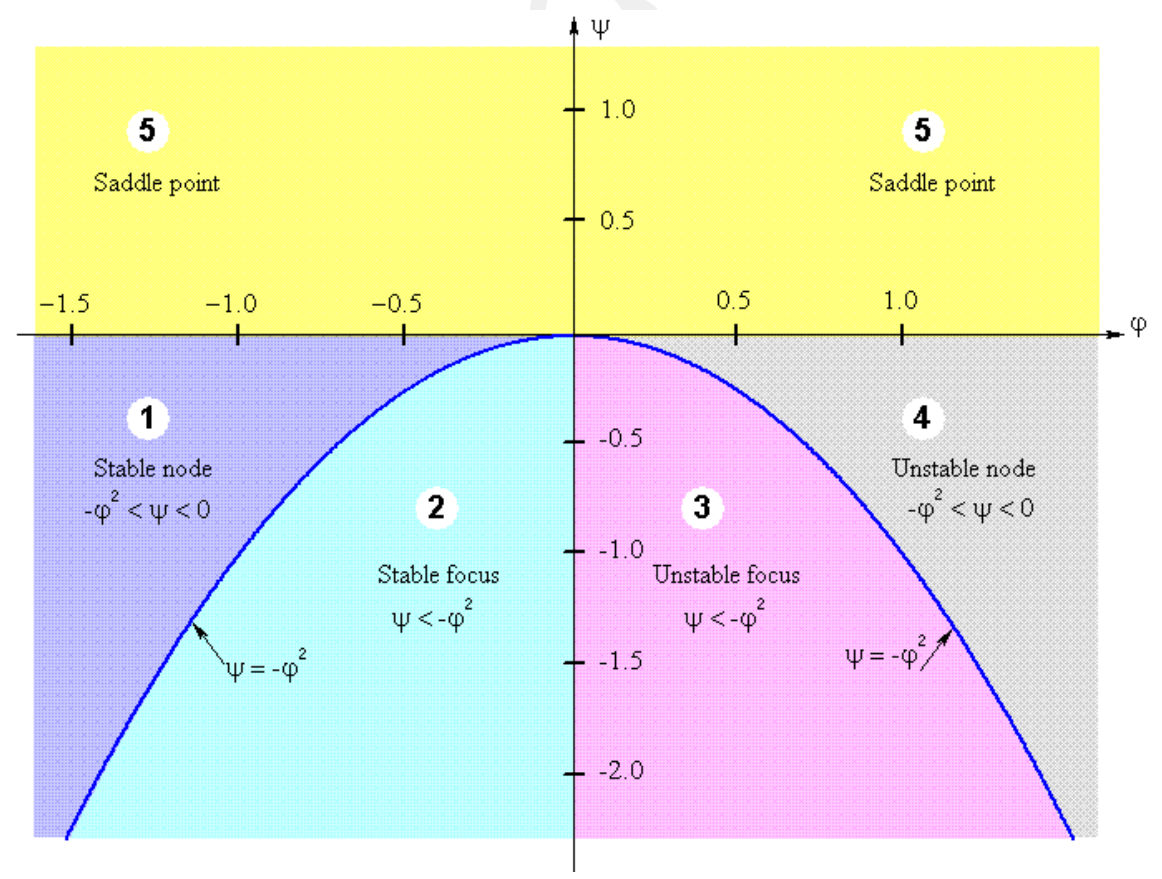

Figure 3.1: Stability / instability domains of the neutral state in the $\varphi / \psi$ plane

The state $(x, y)=(0,0)$ is 
1. a stable node if the roots are real and both negative,

2. a stable focus if the roots are complex with negative real part,

3. an unstable focus if the roots are complex with positive real part,

4. an unstable node if the roots are real and both positive and

5. a saddle point if the roots are real and one is positive.

These five cases lead to the following conditions for $\varphi$ and $\psi$ and thereafter for the trend parameters $\{\kappa, \gamma, \beta, \mu, \nu\}$.

$\begin{array}{rrrl}\text { 1. } & \varphi<0 & -\varphi^{2}<\psi<0 \\ \text { 2. } & \varphi<0 & \psi<-\varphi^{2} \\ \text { 3. } & \varphi>0 & \psi<-\varphi^{2} \\ \text { 4. } & \varphi>0 & -\varphi^{2}<\psi<0 \\ \text { 5. } & -\infty<\varphi<+\infty & \psi>0 .\end{array}$

Figure 3.1 shows the five domains in the $\varphi / \psi$ plane and shows that below the parabola $\psi=-\varphi^{2}$ the neutral state is a focus and above a node or a saddle point.

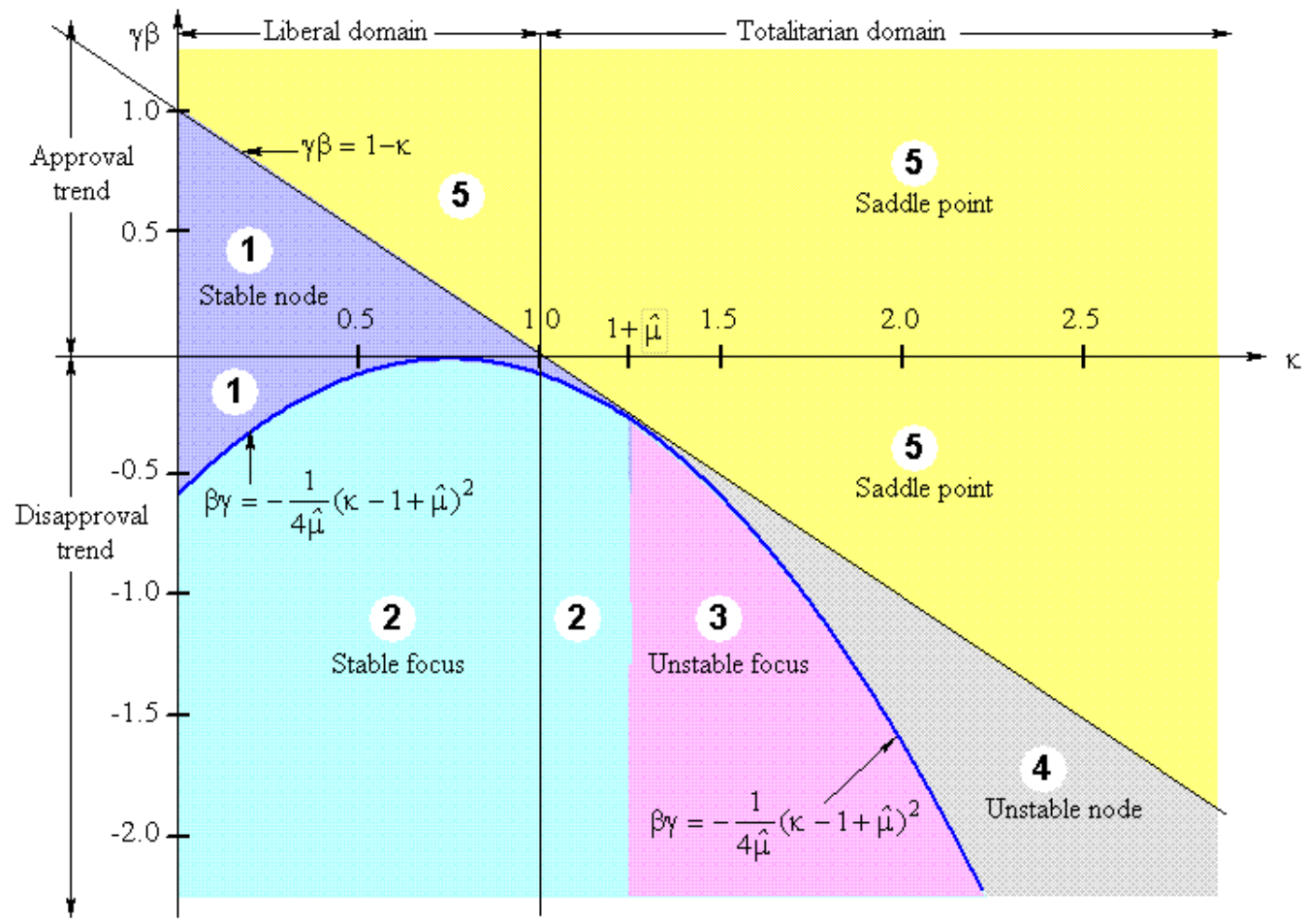

Figure 3.2: Stability / instability domains of the neutral state in the $\kappa / \gamma \beta$ plane for $\hat{\mu}=\frac{\mu}{\nu}=\frac{1}{4}$

For interpretational purposes it is more instructive to represent the five domains (3.15) in the $\kappa / \gamma \beta$ plane of the original trend parameters and to treat the speed ratio $\hat{\mu}$ each time as a fixed parameter. From the definitions of $\rho, \varphi$ and $\psi$ there 
follows in particular:

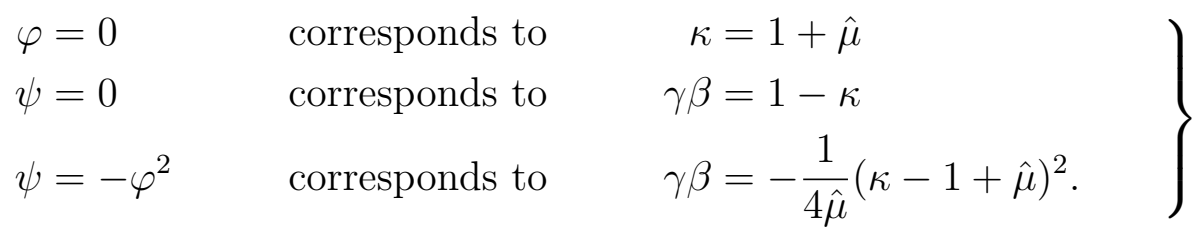

In figures 3.2 and 3.3 the five stability / instability domains (3.15) of the neutral state are shown in the $\kappa / \gamma \beta$ plane for the parameters $\hat{\mu}=\frac{1}{4}\left(\rightarrow \mu=\frac{1}{5}\right)$ and $\hat{\mu}=\frac{1}{16}\left(\rightarrow \mu=\frac{1}{17}\right)$.

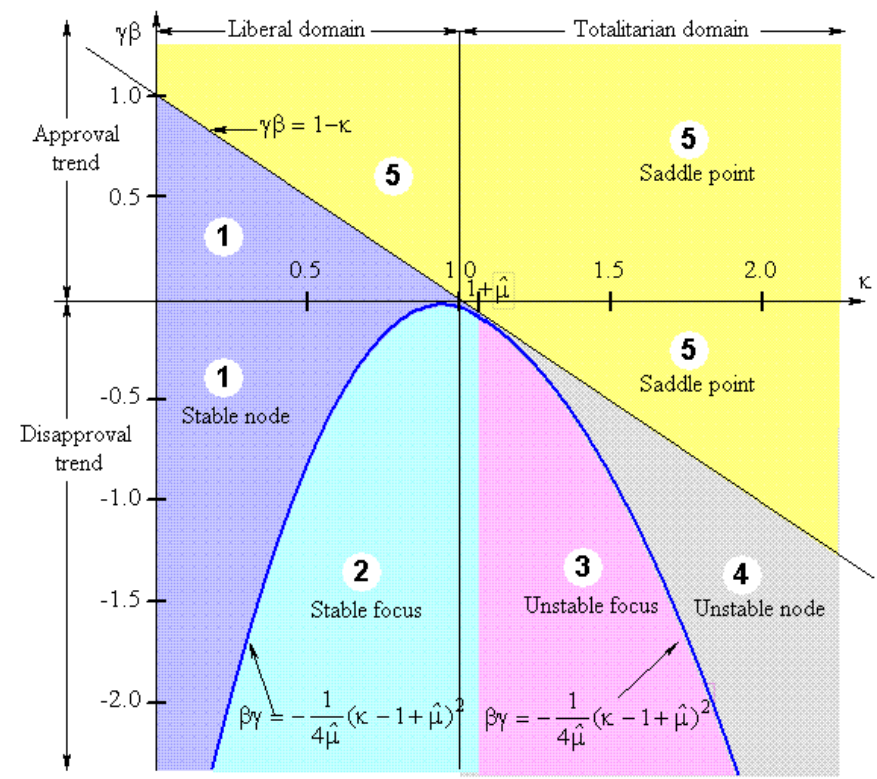

Figure 3.3: Stability / instability domains of the neutral state in the $\kappa / \gamma \beta$ plane for $\hat{\mu}=\frac{\mu}{\nu}=\frac{1}{16}$

The four quadrants in the $\kappa / \gamma \beta$ plane are, in counter-clock-sense,

$\begin{array}{lll}\text { quadrant A } & \kappa>1 & \gamma \beta>0 \\ \text { quadrant B } & 0<\kappa<1 & \gamma \beta>0 \\ \text { quadrant C } & 0<\kappa<1 & \gamma \beta<0 \\ \text { quadrant D } & \kappa>1 & \gamma \beta<0 .\end{array}$

They now comprise different stability / instability domains of the neutral state:

- quadrant A saddle points only,

- quadrant B saddle points and stable nodes,

- quadrant $\mathrm{C}$ stable nodes and stable foci,

- quadrant D stable and unstable foci, unstable nodes and saddle points.

Concerning the socio-political interpretation it will be shown in section 3.7 that the quadrants belong to the following main characteristics:

- quadrant A, totalitarian regime with approval trend 
- quadrant B, liberal regime with approval trend

- quadrant $\mathrm{C}$, liberal regime with disapproval trend

- quadrant $\mathrm{D}$, totalitarian regime with disapproval trend

\subsection{Equilibrium Lines, Stationary States, Slopes at $(0,0)$}

To get more insight into the global dynamics of the system we discuss in this section the equilibrium lines. They are defined by those lines in the $x / y$ plane where one variable comes to rest. This is the case for $\frac{d x}{d \tau}=0$ or $\frac{d y}{d \tau}=0$. The equilibrium lines are denoted by $x_{E}(y)$ and $y_{E}(x)$.

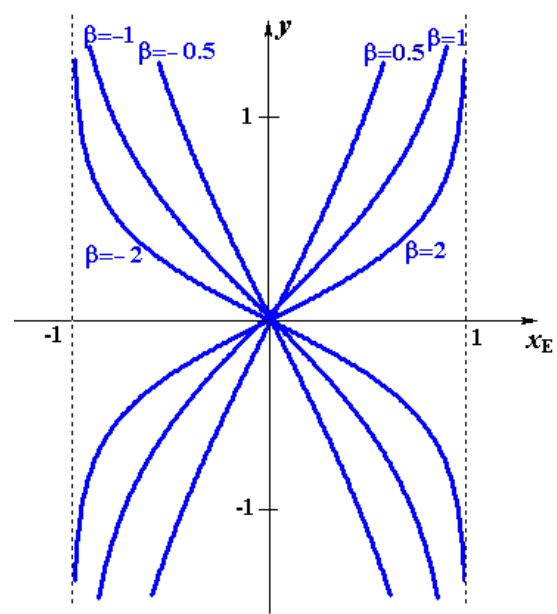

Figure 3.4: Equilibrium function $x=x_{E}(y ; \beta)$ for different values of $\beta$

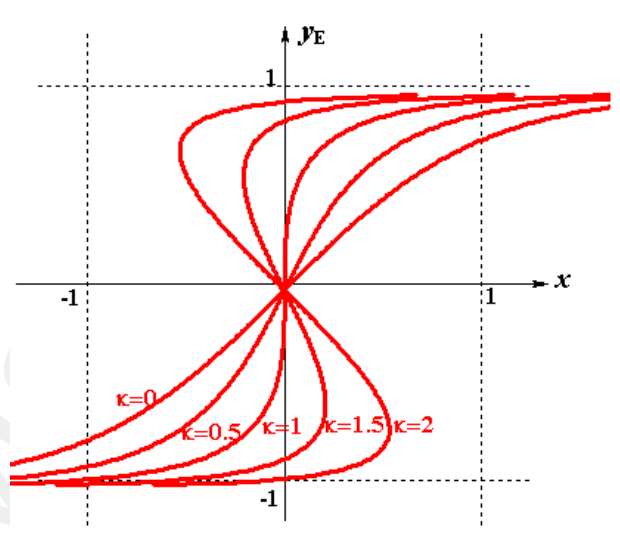

Figure 3.5: Equilibrium function $y=y_{E}(x ; \kappa)$ for $\gamma=1$ and different values of $\kappa$

\section{Equilibrium Line $x_{E}(y ; \beta)$}

From (3.2) we get for $x_{E}(y ; \beta)$

$$
\frac{d x}{d \tau}=\mu\{(1-x) \exp (\beta y)-(1+x) \exp (-\beta y)\}_{x=x_{E}}=0
$$

This gives for $x$

$$
x=x_{E}(y ; \beta)=\tanh (\beta y) .
$$

Solving for $y$

$$
y=y\left(x_{E} ; \beta\right)=\frac{1}{\beta} \operatorname{arctanh}\left(x_{E}\right)=\frac{1}{2 \beta} \ln \left(\frac{1+x_{E}}{1-x_{E}}\right) .
$$

Equilibrium Line $y=y_{E}(x ; \kappa, \gamma)$

From (3.3) we get for $y=y_{E}(x ; \kappa, \gamma)$

$$
\frac{d y}{d \tau}=\nu\{(1-y) \exp (\kappa y+\gamma x)-(1+y) \exp (-\kappa y+\gamma x)\}_{y=y_{E}}=0 .
$$


This gives for $y$

$$
y=y_{E}(x ; \kappa, \gamma)=\tanh \left(\kappa y_{E}+\gamma x\right) .
$$

Solving for $x$,

$$
x=\frac{1}{\gamma}\left\{\operatorname{arctanh}\left(y_{E}\right)-\kappa y_{E}\right\}=\frac{1}{\gamma}\left\{\frac{1}{2} \ln \left(\frac{1+y_{E}}{1-y_{E}}\right)-\kappa y_{E}\right\} .
$$

Figure 3.5 shows $y_{E}(x ; \kappa, \gamma)$ for different values of $\kappa$ and for $\gamma=1$. For $\gamma>1$ the graphs are shrinked and for $\gamma<1$ stretched in $x$ direction.

It turns out that $y_{E}(x ; \kappa, \gamma)$ is

- for $0<\kappa<1$ a unique function of $x$

- for $\kappa>1$ a unique function of $x$ only for large values of $|x|$ and a three-valued function of $x$ for smaller values of $|x|$

Values with $|x|>1$ cannot be reached by the dynamics of the system.

Stationary states $\left(x_{S}, y_{S}\right)$

Stationary states $\left(x_{S}, y_{S}\right)$ are defined as those points in $(x, y)$ plane where both variables $x(\tau)$ and $y(\tau)$ come to rest. This means that

$$
\left.\frac{d x}{d \tau}\right|_{x_{S}, y_{S}}=0 \quad \text { and }\left.\quad \frac{d y}{d \tau}\right|_{x_{S}, y_{S}}=0
$$

must hold simultaneously. Thus stationary states are given by the intersection point of the equilibrium lines $x_{E}$ and $y_{E}$ given by the equations

$$
y_{S}=\tanh \left(\kappa y_{S}+\gamma x_{S}\right) \text { and } x_{S}=\tanh \left(\beta y_{S}\right) .
$$

The neutral state $\left(x_{S}, y_{S}\right)=(0,0)$ is one stationary state. Depending on the trend parameters $(\kappa, \gamma, \beta)$ there may exist further stationary states. (A dependence on $\hat{\mu}$ does not exist as $\hat{\mu}$ is not included in (3.24).)

We get more insight by considering the slopes of the equilibrium lines at the neutral state. They are determined from the defining equations (3.19) and (3.21). We denote the derivative of the equilibrium line $y_{E}$ by $f_{y_{E}}^{\prime}$ and the derivative of $x_{E}$ by $f_{x_{E}}^{\prime}$ :

$$
\begin{aligned}
& \left.f_{x_{E}}^{\prime}\right|_{(0,0)}=\left.\frac{d y}{d x_{E}}\right|_{(0,0)}=1 /\left.\frac{d x_{E}(y ; \beta)}{d y}\right|_{(0,0)}=\frac{1}{\beta} \\
& \left.f_{y_{E}}^{\prime}\right|_{(0,0)}=\left.\frac{d y_{E}}{d x}\right|_{(0,0)}=\left.\frac{d y_{E}(x ; \kappa, \gamma)}{d x}\right|_{(0,0)}=\frac{\gamma}{(1-\kappa)} .
\end{aligned}
$$

The domain of definition of the trend parameters is given in equation (2.23). As $0 \leq \kappa<\infty ; \quad 0<\gamma<\infty ; \quad-\infty<\beta<+\infty$, both slopes can assume all values from $-\infty$ to $+\infty$. 
Depending on the form of the equilibrium lines, there may exist in addition to the neutral state either zero, two or four stationary states. They belong to the different quadrants of the trend parameter plane (figures 3.2 and 3.3).

\begin{tabular}{|c|c|c|c|c|}
\hline & $\begin{array}{c}\text { slopes at } \\
(\mathbf{0 , 0})\end{array}$ & trend parameters & quadrant & $\begin{array}{c}\text { additional station- } \\
\text { ary states }\end{array}$ \\
\hline 1. & $f_{y_{E}}^{\prime}>f_{x_{E}}^{\prime}>0$ & $\gamma \beta>(1-\kappa)>0$ & $\mathrm{~B}$ & 2 \\
\hline 2. & $f_{y_{E}}^{\prime}>0>f_{x_{E}}^{\prime}$ & $(1-\kappa)>0>\gamma \beta$ & $\mathrm{C}$ & 0 \\
\hline 3. & $0>f_{y_{E}}^{\prime}>f_{x_{E}}^{\prime}$ & $0>\gamma \beta>(1-\kappa)$ & $\mathrm{D}$ & 2 \\
\hline 4. & $f_{x_{E}}^{\prime}>f_{y_{E}}^{\prime}>0$ & $(1-\kappa)>\gamma \beta>0$ & $\mathrm{~B}$ & 0 \\
\hline 5. & $f_{x_{E}}^{\prime}>0>f_{y_{E}}^{\prime}$ & $\gamma \beta>0>(1-\kappa)$ & $\mathrm{A}$ & 2 \\
\hline 6. & $0>f_{x_{E}}^{\prime}>f_{y_{E}}^{\prime}$ & $0>(1-\kappa)>\gamma \beta$ & $\mathrm{D}$ & 0 or 4 \\
\hline
\end{tabular}

It is evident that the slope relation of the equilibrium lines at the neutral state or the corresponding relation between the trend parameters $\gamma \beta$ and $(1-\kappa)$ already determines the existence (or nonexistence) of further stationary states and therefore the global fate of the system during its evolution with time.

\subsection{The Cusp-Curve}

The equilibrium line $y=y_{E}(x ; \kappa, \gamma)$ is for $\kappa \leq 1$ a unique function of $x$. For $\kappa>1$ it is unique only for $|x|>x_{c}(\kappa, \gamma)$ and a one-to-three function for $|x|<x_{c}$. The one-to-three domain $\mathbb{D}_{3}$ where $y_{E}(x ; \kappa, \gamma)$ has the three values $y_{E+}, y_{E 0}, y_{E-}$ for one $x$ value is separated from the domain $\mathbb{D}_{1}$ where $y_{E}$ is a one-to-one function by $x= \pm x_{c}$. The $y_{E}$ value (with the vertical tangent line) corresponding to $x_{c}$ is $y_{c}$. $\left(x_{c}, y_{c}\right)$ are points of the so-called cusp-curve. From equation (3.22) we get

$$
\begin{aligned}
& \left.\frac{d x\left(y_{E} ; \kappa, \gamma\right)}{d y_{E}}\right|_{y_{E}=y_{c}}=\frac{1}{\gamma}\left\{\frac{1}{1-y_{c}^{2}}-\kappa\right\}=0 \\
& \text { or } \quad y_{c}(\kappa)= \pm \sqrt{\frac{\kappa-1}{\kappa}},
\end{aligned}
$$

showing that real values for $y_{c}(\kappa)$ exist only for $\kappa>1$.

Inserting $y_{c}$ into equation (3.22) we get the corresponding value $x_{c}$ :

$$
\begin{aligned}
x_{c}(\kappa, \gamma) & =\frac{1}{\gamma}\left\{\frac{1}{2} \ln \frac{1+y_{c}(\kappa)}{1-y_{c}(\kappa)}-\kappa y_{c}(\kappa)\right\} \\
& =\mp \frac{1}{\gamma}\{\sqrt{\kappa(\kappa-1)}-\ln (\sqrt{\kappa}+\sqrt{\kappa-1})\} .
\end{aligned}
$$

We remark that positive (negative) values of $y_{c}(\kappa)$ belong to negative (positive) values of $x_{c}(\kappa)$. This will be essential for the interpretation in terms of catastrophe theory following in section 4 . 


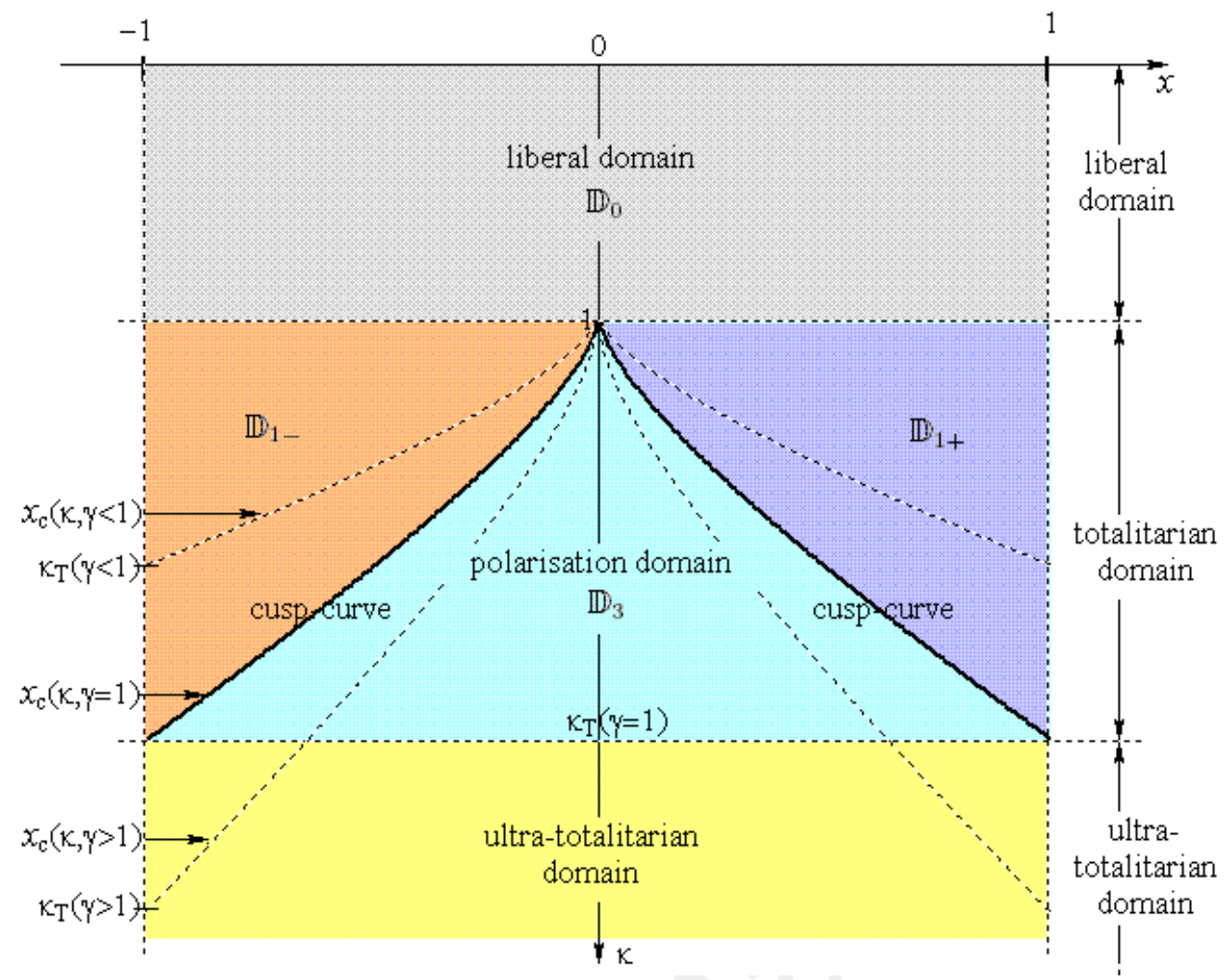

Figure 3.6: Cusp-Curves $x=x_{c}(\kappa, \gamma)$ in $x, \kappa$ plane for different values of $\gamma$

Figure 3.6 shows the projection of the cusp-curve $x=x_{c}(\kappa, \gamma)$ in the $x, \kappa$ plane for different values of $\gamma$. In each case the cusp-curve separates the domains $\mathbb{D}_{3}$ and $\mathbb{D}_{1}$.

We also see that $x=x_{c}(\kappa, \gamma)$ is for $x>0$ a monotonous function of $\kappa$. The same holds for $x<0$. With increasing $\kappa$ the boundaries of the $x$-domain $x= \pm 1$ are reached. The corresponding $\kappa$ value is $\kappa_{T}$. From equation (3.29) $\kappa_{T}$ is defined by

$$
\sqrt{\kappa_{T}\left(\kappa_{T}-1\right)}-\ln \left\{\sqrt{\kappa_{T}}+\sqrt{\kappa_{T}-1}\right\}=\gamma .
$$

At $\left(x_{c}= \pm 1, \kappa_{T}\right)$ the cusp-curve leaves the allowed domain $|x| \leq 1$ so that these values of $x_{c}\left(\kappa_{T}, \gamma\right)$ can never be realized by the dynamics of the system. The interpretation will be given in scenario 10 .

\subsection{Flux lines for Selected Scenarios}

One possibility of representing the evolution of a system are its flux lines. They arise by eliminating the time variable from the original differential equations. In our case we obtain from equations (3.2) and (3.3) the differential equation for the flux lines:

$$
\frac{d y / d \tau}{d x / d \tau}=\frac{d y}{d x}=\frac{\nu\{(1-y) \exp (\kappa y+\gamma x)-(1+y) \exp (-(\kappa y+\gamma x))\}}{\mu\{(1-x) \exp (\beta y)-(1+x) \exp (-\beta y)\}} .
$$

The equilibrium lines $x=x_{E}(y ; \beta)$ and $y=y_{E}(x ; \kappa, \gamma)$ are helpful in constructing the flux lines: 
- the line $y=y_{E}(x ; \kappa, \gamma)$ must be crossed horizontally by the flux lines and

- the line $x=x_{E}(y ; \beta)$ must be crossed vertically by the flux lines.

This is obvious as the nominator of the right hand side of equation (3.31) vanishes at $y_{E}$ and the denominator at $x_{E}$. There are different sets of flux lines belonging to the same equilibrium lines. The reason is that the equilibrium lines depend only on $\{\kappa, \gamma, \beta\}$ whereas the flux lines depend on $\nu / \mu$ (equation (3.31))as well.

As we cannot select an infinite set of flux lines belonging to the infinite manifold of trend parameters $\{\kappa, \gamma, \beta, \nu, \mu\}$, we use the following ordering principles to select qualitatively different and characteristic scenarios:

1. The quadrants of the trend parameter plane

- A: totalitarian / approving

- B: liberal / approving

- C: liberal / disapproving

- D: totalitarian / disapproving

run through in anti-clockwise-direction,

2. the parameter $\mu$ determining the evolution speed of $x(\tau)$ and $y(\tau)$,

3. the status of the neutral state being a stable node or focus, an unstable node, focus or saddle point,

4. the order of the slopes of the equilibrium lines at the neutral state leading to different numbers of stationary states.

The selected scenarios are listed in the following table and in figure 3.7.

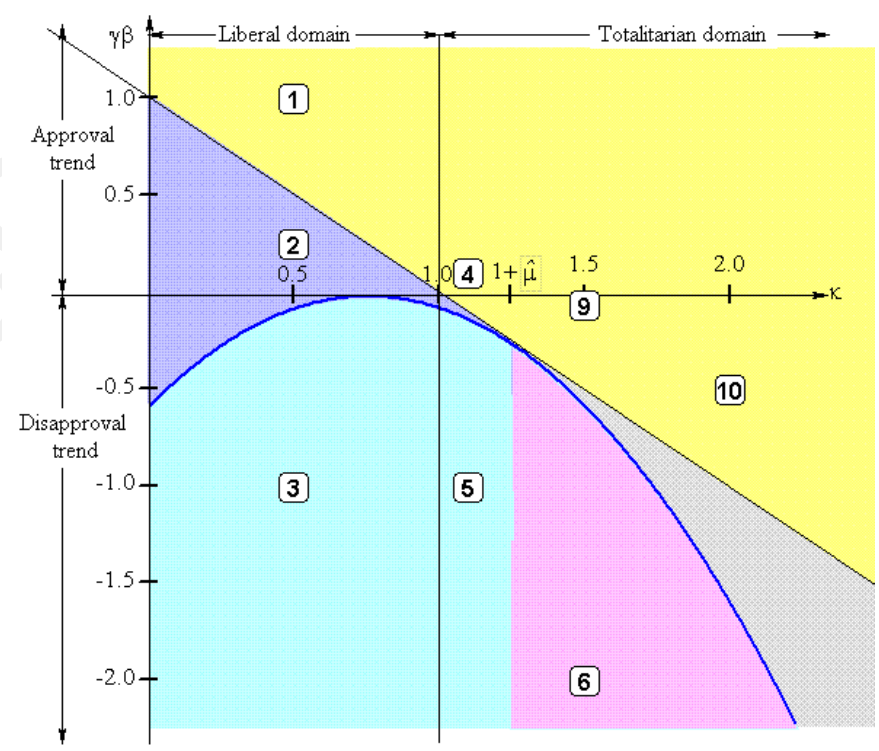

Figure 3.7: Position of the different scenarios with $\mu=0.2$ in the 4 quadrants 


\begin{tabular}{|c|c|c|c|c|c|c|c|c|}
\hline 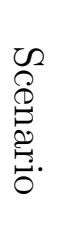 & 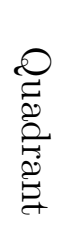 & $\kappa$ & $\gamma$ & $\beta$ & $\mu$ & $\begin{array}{l}\text { Status of neu- } \\
\text { tral state }\end{array}$ & Slope relations & $\begin{array}{l}\text { Number of } \\
\text { additional } \\
\text { stationary } \\
\text { states }\end{array}$ \\
\hline 1 & B & 0.5 & 1 & 1 & 0.2 & unstable saddle & $f_{y_{E}}^{\prime}>f_{x_{E}}^{\prime}>0$ & 2 \\
\hline 2 & B & 0.5 & 0.25 & 1 & 0.2 & stable node & $f_{x_{E}}^{\prime}>f_{y_{E}}^{\prime}>0$ & 0 \\
\hline 3 & $\mathrm{C}$ & 0.5 & 1 & -1 & 0.2 & stable focus & $f_{y_{E}}^{\prime}>0>f_{x_{E}}^{\prime}$ & 0 \\
\hline 4 & A & 1.1 & 0.5 & 0.2 & 0.2 & unstable saddle & $f_{x_{E}}^{\prime}>0>f_{y_{E}}^{\prime}$ & 2 \\
\hline 5 & $\mathrm{D}$ & 1.1 & 1 & -1 & 0.2 & stable focus & $0>f_{x_{E}}^{\prime}>f_{y_{E}}^{\prime}$ & 0 \\
\hline 6 & $\mathrm{D}$ & 1.5 & 1 & -2 & 0.2 & unstable focus & $0>f_{x_{E}}^{\prime}>f_{y_{E}}^{\prime}$ & 0 \\
\hline 7 & $\mathrm{D}$ & 1.5 & 1 & -2 & 0.05 & unstable focus & $0>f_{x_{E}}^{\prime}>f_{y_{E}}^{\prime}$ & 0 \\
\hline 8 & $\mathrm{D}$ & 2.0 & 1 & -1.2 & 0.02 & unstable node & $0>f_{x_{E}}^{\prime}>f_{y_{E}}^{\prime}$ & 0 \\
\hline 9 & $\mathrm{D}$ & 1.5 & 0.25 & -0.25 & 0.2 & unstable saddle & $0>f_{y_{E}}^{\prime}>f_{x_{E}}^{\prime}$ & 2 \\
\hline 10 & $\mathrm{D}$ & 2.0 & 0.5 & -1 & 0.2 & unstable saddle & $0>f_{x_{E}}^{\prime}>f_{y_{E}}^{\prime}$ & 4 \\
\hline
\end{tabular}

\subsection{Socio-political Interpretation of the Selected Scenarios}

We give now an interpretation of the collective evolution of the societies whose "political moods or climates" are characterized by the trend parameters given in the table "Selected Scenarios". In doing so we take into account the role of the trend parameters explained in section 2.5.

\section{Scenario 1}

$\kappa \longdiv { 0 . 5 } \gamma \longdiv { 1 } \beta \longdiv { 1 } \mu \longdiv { 0 . 2 0 0 }$

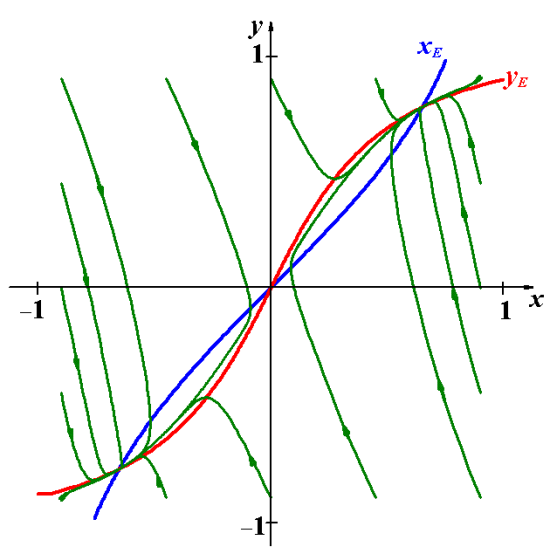

This society is rather liberal $(\kappa=0.5)$. The inner inclination of people has a rather strong influence on the majority formation $(\gamma=1)$. People tend to approve the ruling political line $(\beta=1)$. The inner inclination is moderately steadfast, slowly moving with $(\mu=0.2)$. Due to the approval trend $(\beta=1)$ the neutral state destabilizes (saddle) and a moderate publicly exhibited majority $\left(y_{S}\right)$ for the ruling political line stabilizes together with moderate affirmative inner inclination $\left(x_{S}\right)$. It depends on initial conditions whether $y_{S}>0, x_{S}>0$ or $y_{S}<0, x_{S}<0$

is approached. 


\section{Scenario 2}

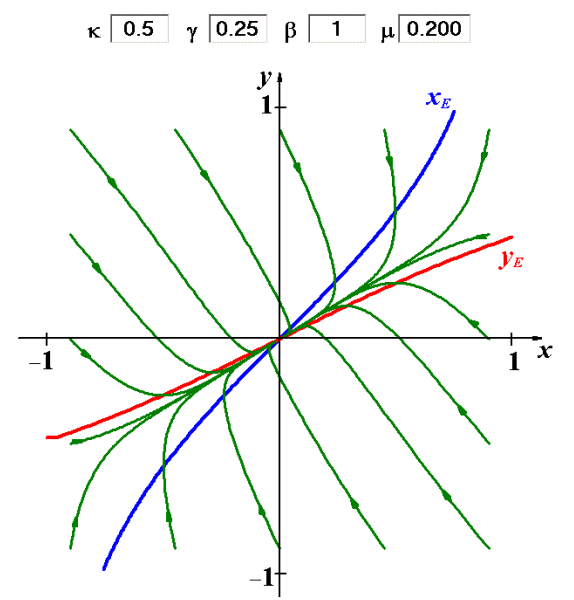

\section{Scenario 3}

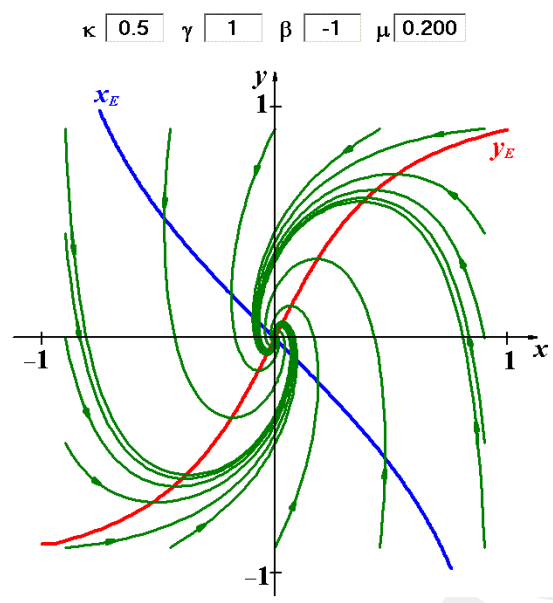

ernable".

\section{Scenario 4}

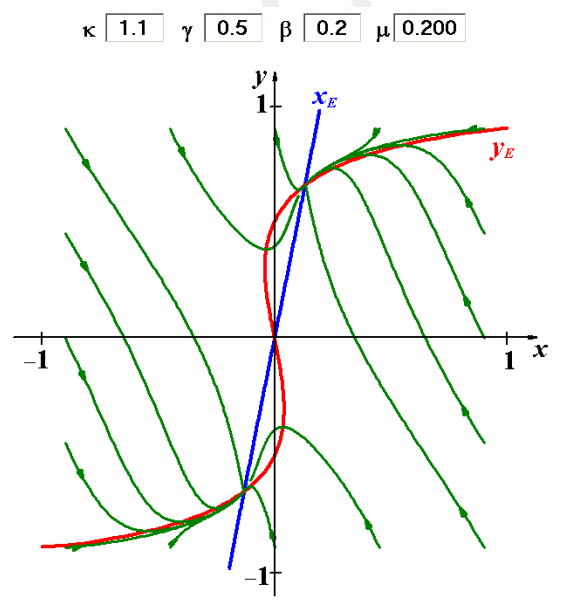

This society is rather liberal $(\kappa=0.5)$. Although people tend to approve the existing political line $(\beta=1)$, they are "unpolitical" and therefore have a weak influence $(\gamma=0.25)$ on the majority formation only . The inner inclination moves slowly with $\mu=0.2$. Under these circumstances the indifferent balanced state of majority $\left(y_{S}=0\right)$ and inner inclination $\left(x_{S}=0\right)$ stabilizes and becomes a stable node.

This society is also rather liberal $(\kappa=0.5)$. However its political climate differs from scenario 2 . The influence of the inner inclination of people on the collective political line is rather strong $(\gamma=1)$, but people tend to disapprove all establishing majorities $(\beta=-1)$. Thus always a steadfast inner opposition arises (with $\mu=0.2$ ). This process thwarts the formation of stabilizing majorities and the neutral state $\left(x_{S}, y_{S}\right)=(0,0)$ is approached in oscillating manner (i. e. the neutral state is a stable focus). Politicians may tend to say "this society is liberal but not gov-

This society is in a moderate totalitarian state $(\kappa=1.1)$. Nevertheless it tends to approve the governing ideology $(\beta=0.2)$, but the inner inclination of people has a weak influence only $(\gamma=$ $0.5)$. The inner inclination is steadfast, slowly moving with $(\mu=0.2)$. The result of those trends is the instability of the neutral state (saddle) and a straight evolution towards a stable relatively high publicly exhibited majority $\left(y_{S}\right)$ and simultaneously to a stable but weak affirmative inner inclination $\left(x_{S}\right)$. It depends on (more or less accidental) initial conditions whether the ideology "+" with $y_{S}>0, x_{S}>0$ or the ideology " -" with $y_{S}<0, x_{S}<0$ is stabilizing. 
The first four scenarios belong to quadrants $\mathrm{A}, \mathrm{B}$ and $\mathrm{C}$ of the trend parameter plane. The following six scenarios belong to quadrant $\mathrm{D}$ with totalitarian but disapproving societies. They comprise the dramatic cases!

\section{Scenario 5}

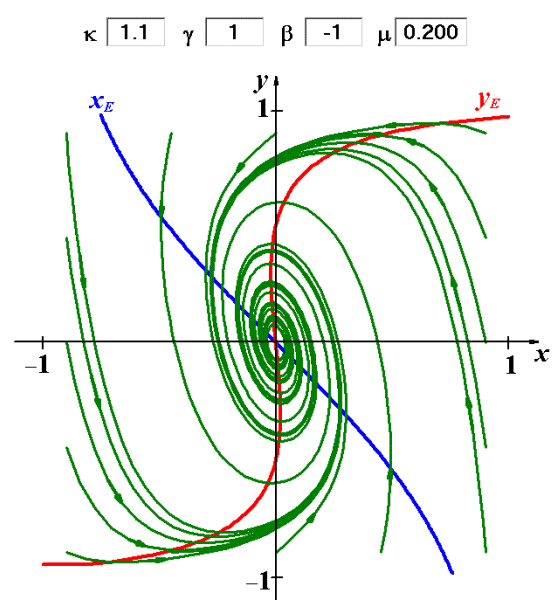

This society is also in a moderate totalitarian state $(\kappa=1.1)$. However the influence of the inner inclination of the people on the established political ideology is rather strong $(\gamma=1)$. On the other hand the disapproving trend against each arising ideology is also strong $(\beta=-1)$. The result is that the neutral state $\left(x_{S}, y_{S}\right)=$ $(0,0)$ is still a stable focus, but it is approached only slowly $(\mu=0.2)$ under large oscillations of the majority variable $y$ (between $y>0$ and $y<$ 0 ) and the inner inclination $x$ (between $x>0$ and $x<0)$.

\section{Scenario 6}

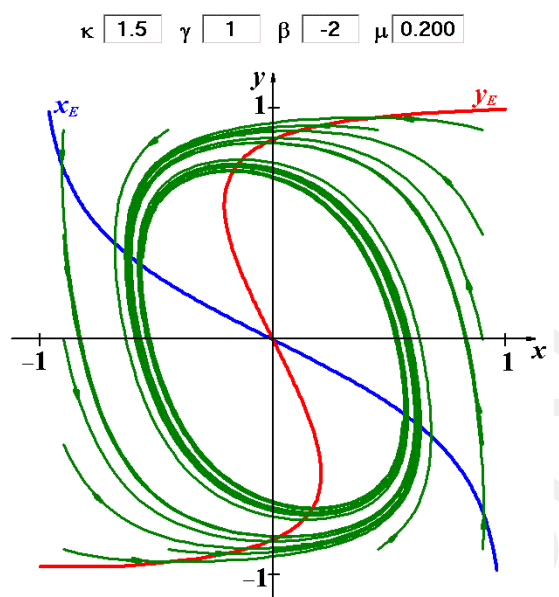

This society is in a strongly developed totalitarian state with high opinion pressure $(\kappa=1.5)$ exceeding the attempt of people to take influence $(\gamma=1)$. Due to opinion pressure a long lasting majority $y>0$ for the political ideology "+" stabilizes at first. This ideology destabilizes the neutral state, which is now an unstable focus. On the other hand there exists a very strong disapproval trend $(\beta=-2)$ drawing the inner inclination slowly but steadfastly $(\mu=0.2)$ towards a strong opposition against ideology " +". If the inner dissidence $x<0$ becomes strong and influential enough, the ideology of the majority eventually breaks down. Then the opposite ideology " -" gains momentum in a "revolutionary" transient period and develops a strong majority $y<0$. This majority $y<0$ is at first stabilized by the persisting opinion pressure. However people have kept their disapproval trend $(\beta=-2)$, now drawing their inner inclination slowly but steadfastly towards strong inner opposition, this time against ideology "-". The breakdown of this ideology follows. A revival of ideology "+" will happen since the high opinion pressure persists. The whole cyclic process approaches a limit cycle under the assumed condition that the parameters $\kappa, \gamma, \beta, \mu$ remain constant. 


\section{Scenario 7}

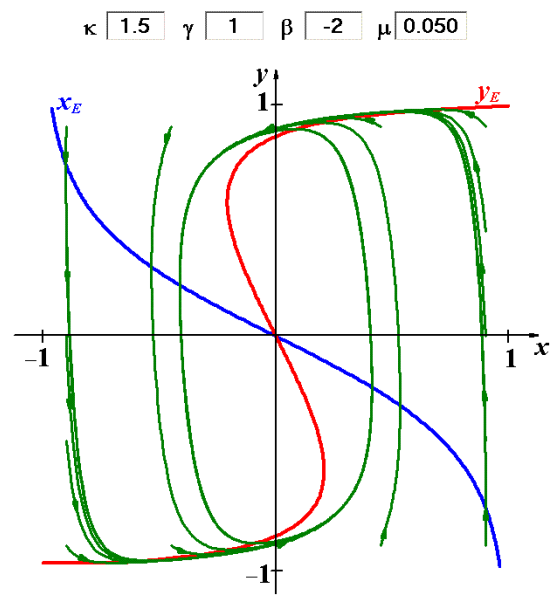

This society has the same trend parameters $\kappa=$ $1.5, \gamma=1, \beta=-2$ as society 6 . It is strongly totalitarian, the opinion pressure $(\kappa=1.5)$ exceeding people's attempt to take influence $(\gamma=1)$ with strong disapproval trend $(\beta=-2)$. The equilibrium lines $x_{E}$ and $y_{E}$ depending only on $\kappa, \gamma, \beta$ also agree with those of scenario 6 . The only difference to scenario 6 is the small speed parameter $\mu=0.05$. Therefore the very steadfast inner inclination goes into opposition with a very slow speed. This leads to differences in the flux lines and in the manner how stagnant and revolutionary phases of the limit cycle are traversed. The breakdown of the majority $y>0$ for ideology " + " follows soon after $x<0$ has passed the value $x_{c}(\kappa)$ of the cusp-curve.

\section{Scenario 8}

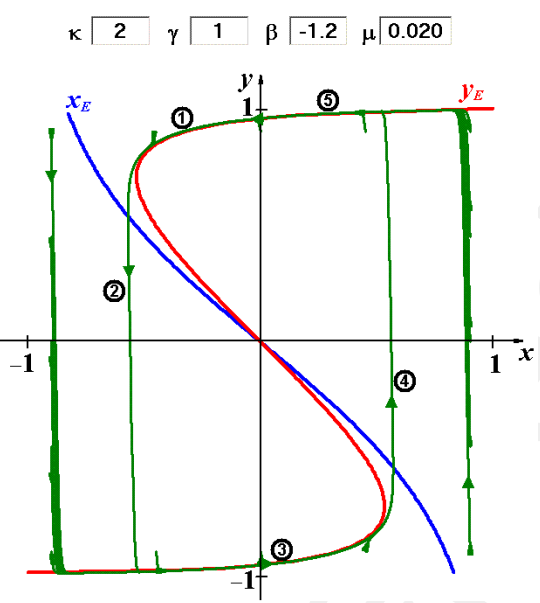

This society is a totalitarian society with very high opinion pressure $\kappa=2.0$ exceeding people's attempt to take influence $(\gamma=1)$. The disapproval trend $\beta=-1.2$ is strong, but not as strong as in scenarios 6 and 7 . The neutral state is now an unstable node instead of an unstable focus. This means that initially a majority $y>0$ (or $y<0$ depending on initial conditions) is reached more directly than in the previous scenario 6 and 7. However the disapproval trend $(\beta=-1.2)$ is strong enough to let this majority break down. Again there evolves a limit cycle scenario similar to that of cases 6 and 7 . As the speed parameter is now very slow $(\mu=0.02)$, the breakdown follows immediately after a sufficient high amplitude of dissident inner inclination $x<0$ has developed and crossed the cusp-curve $x_{c}(\kappa)$. The movement in $x$-direction is proportional to $\mu$ and that in $y$-direction proportional to $(1-\mu)$ (equations (3.2) and (3.3)). Therefore the movement along the equilibrium line $y_{E}$ is very slow whereas the breakdown at the cusp-curve occurs with an extremely high speed. This behavior will be dramatic if we are approaching the limit $\mu \rightarrow 0$. The case of $\mu \rightarrow 0$ together with the different stages 1 to 5 shown in the figure can be treated in terms of the concepts of catastrophe theory. We will do this in section 4 . 


\section{Scenario 9}

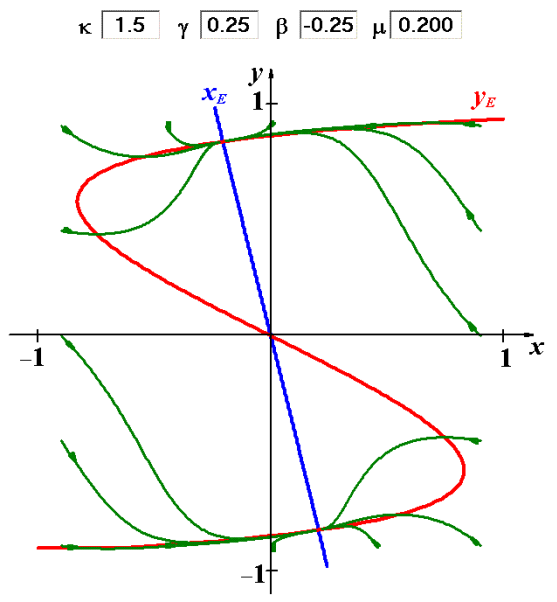

This society is "the dictators and his supporters hope and simultaneously fear". It is only stable by pressure maintained against a dissident population. A stronger disapproval trend $\beta<0$ could lead to the breakdown of the majority as in the scenarios 6, 7 and 8. It is totalitarian with high opinion pressure $(\kappa=1.5)$. The people's inner inclination has a weak influence $(\gamma=0.25)$ on the collective majority formation only, and people have a weak disapproval trend $(\beta=-0.25)$ as well. The neutral state is an unstable saddle, and due to the opinion pressure there develops a "self-organizing" collective majority $y>0$ (or $y<0$ depending on the initial conditions). The weak disapproval trend $(\beta=-0.25)$ is not sufficient to develop a disinclination amplitude $x<0$ strong enough to destabilize the majority $y>0$. Therefore eventually a stationary state $\left(y_{S}>0, x_{S}<0\right)$ is reached where $y_{S}>0$ is a strong majority stabilized by opinion pressure only and $x_{S}<0$ is a state of inner dissidence too weak to destabilize $y_{S}$.

\section{Scenario 10}

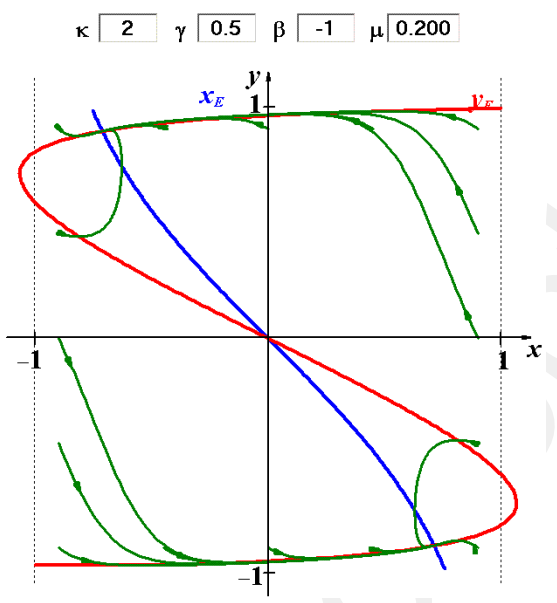

This society is ultra totalitarian. Its opinion pressure $\kappa=2>\kappa_{T}(\gamma)$ is extremely high and exceeds the value $\kappa_{T}(\gamma)$ (see figure 3.6) whereas the inclination influence is low $(\gamma=0.5)$. The cusp-curve $x_{c}(\kappa, \gamma)$ is now outside the domain of definition $|x| \leq 1$. In spite of a strong disapproval trend $(\beta=-1)$ the flux lines can never cross the cuspcurve. A stationary state either $\left(y_{S}>0, x_{S}<0\right)$ or $\left(y_{S}<0, x_{S}>0\right)$ is eventually reached, similar to that of scenario 9 . This society is "the dictator's dream" because for the given values $\kappa>\kappa_{T}(\gamma)$ and $\gamma$ the dynamics of the variables $x(\tau)$ and $y(\tau)$ can never lead to a breakdown of the totalitarian regime, even if $\beta$ grows to more extreme negative values.

What is then "the people's hope", that is the hope to get rid of the established totalitarian regime? The two possibilities remain:

- The inner socio-political state of the system can change. This can happen by change of the trend parameters, for example by reducing $\kappa$ through more reason and less ideology within the elites of the regime (i. e. reform from above) or by increasing the inclination influence $\gamma$. This increase could lead from $\kappa_{T}(\gamma)<\kappa$ to $\kappa_{T}(\gamma)>\kappa$ (i.e. back from scenario 10 to scenarios 6 or $7)$. 
- The more risky (but perhaps unavoidable) way with indefinite result is an interference from outside with the purpose of "regime change". This possibility is beyond our model design. In any case a weighing of chances and risks of this way should take place beforehand.

\section{The Limit of Catastrophe Theory}

Two of the scenarios comprised within our model, namely scenario 7 and 8, exhibit almost discontinuous changes between stagnant and revolutionary phases. Discontinuous behavior is, on the other hand, central to the approach of catastrophe theory. Therefore it is interesting to discuss the relation of both approaches by means of the present model.

The starting point of elementary catastrophe theory (CT) is a mathematical theorem of René Thom. He starts from a set $\mathbf{x}$ of variables $x_{i}(t)$ satisfying the gradient dynamics $d x_{i} / d t=-\partial V(\mathbf{x}, \mathbf{c}) / \partial x_{i}$, where the potential $V$ depends on the dynamic variables $x_{i}$ as well as on constant control parameters $\mathbf{c}$. Now the equilibrium defined by $\partial V(\mathbf{x}, \mathbf{c}) / \partial x_{i}=0$ is considered. It establishes a nonlinear relation between the equilibrium values of $x_{i}$ and the control parameters $\mathbf{c}$. There may belong one or more equilibrium values $\mathbf{x}$ to a given set of $\mathbf{c}$. This means that a discontinuity can occur. In its vicinity the nonlinear relation can be cast into a "canonical" form. For the cases of one or two variables (x) and up to four control parameters (c), Thom thus arrives at the standard form of "seven elementary catastrophes".

Let us now go into details of this limiting case for which our model coincides with catastrophe theory.

\subsection{The Potential Limit}

We can write the differential equations (3.2) and (3.3) in the general form

$$
\frac{d y}{d \tau}=f(y, x ; \nu, \kappa, \gamma) \quad \text { and } \quad \frac{d x}{d \tau}=g(x, y ; \mu, \beta) .
$$

Now we ask whether there exists a global potential $W(y, x ; \kappa, \gamma, \beta, \nu, \mu)$ allowing to write the equation (4.1) in the form

$$
\frac{d y}{d \tau}=-\frac{\partial W}{\partial y} ; \quad \frac{d x}{d \tau}=-\frac{\partial W}{\partial x} .
$$

The necessary and sufficient condition for the existence of such a potential $W$ would be

$$
\frac{d f}{d x}=-\frac{\partial^{2} W}{\partial x \partial y}=\frac{d g}{d y}=-\frac{\partial^{2} W}{\partial y \partial x} .
$$

Evidently this condition for $f(y, x ; \nu, \kappa, \gamma)$ and $g(x, y ; \mu, \beta)$ is not fulfilled. 
However there exists a limit case corresponding also to the important scenarios 7 and 8 of section 3 for which a potential can be constructed. This is the case where the variable $x$ is practically at rest. In this case we put

$$
\mu=\varepsilon ; \quad \nu=1-\varepsilon ; \quad x(\tau) \approx x_{0} ; \quad \text { with } \quad \varepsilon \ll 1 .
$$

Since now $y(\tau)$ is the only dynamical variable one can write

$$
\frac{d y}{d \tau}=f\left(y, x=x_{0} ; \kappa, \gamma, \nu\right)=-\frac{\partial V\left(y, x_{0} ; \kappa, \gamma, \nu\right)}{\partial y}
$$

where the potential $V$ is constructed by

$$
\left.\begin{array}{l}
V\left(y, x_{0} ; \kappa, \gamma, \nu\right)=-\int f\left(y, x_{0} ; \kappa, \gamma, \nu\right) d y+c\left(x_{0}\right), \text { resulting in } \\
V=\frac{2 \nu}{\kappa^{2}}\left\{\kappa y \sinh \left(\kappa y+\gamma x_{0}\right)-(1+\kappa) \cosh \left(\kappa y+\gamma x_{0}\right)\right\}+c\left(x_{0}\right) .
\end{array}\right\}
$$

Here $c\left(x_{0}\right)$ is a constant of integration which may depend on $x_{0}$.

The extrema $y_{E}$ of $V$ are given by

$$
\begin{aligned}
& \left.\frac{\partial V}{\partial y}\right|_{y=y_{E}}=-f\left(y_{E}, x_{0} ; \kappa, \gamma, \nu\right)=0 \\
& \text { leading to } \quad y_{E}=\tanh \left(\kappa y_{E}+\gamma x_{0}\right) .
\end{aligned}
$$

This equation is identical with equation (3.21) of the equilibrium line $y=y_{E}\left(x_{0} ; \kappa, \gamma\right)$ of equation (3.3).

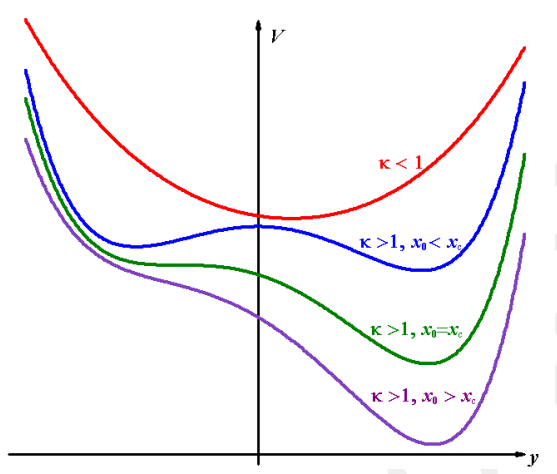

The potential $V$ is a function of $y$ depending on the four parameters $x_{0}, \kappa, \gamma$ and $\nu$. The dependance on $\kappa$ and $x_{0}$ is relevant, whereas the dependance on $\nu$ and $\gamma$ leads only to scaling and shifting effects of $V$ and $y$. The following cases shown in the figure have to be distinguished:

For $\kappa<1$ the potential has one minimum only (domain $\mathbb{D}_{0}$ of figure 3.6).

For $\kappa>1$ and $x_{0}<x_{c}$ the potential has two minima and one maximum in between $\left(\right.$ domain $\mathbb{D}_{3}$ ). With increasing $x_{0}$ the left minimum becomes more shallow and mutates for $x_{0}=x_{c}$ to a point of inflection, whereas the the right minimum becomes deeper.

For $\kappa>1$ and $x_{0}>x_{c}$ only the right minimum survives (domain $\mathbb{D}_{1+}$ ).

The corresponding holds for $x_{0}<0$. In this case the graphs are reflected at the $V$ axes.

In catastrophe theory it is usual to show $y=y_{E}\left(x_{0}, \kappa, \gamma=1\right)$ instead of the potential. $y_{E}$ presents the extrema of $V$ (i. e. $\frac{\partial V}{\partial y}=0$ ). Figure 4.1 shows the area of $y_{E}$ as well as the cusp-curve in the three dimensional space. The analytic form of the cusp-curve was given in equations (3.28) and (3.29). Its projection $\left(x=x_{c}(\kappa)\right)$ and the graphs of $y_{E}(x ; \kappa)$ for discrete values of $\kappa$ were presented in figures 3.5 and 3.6. 


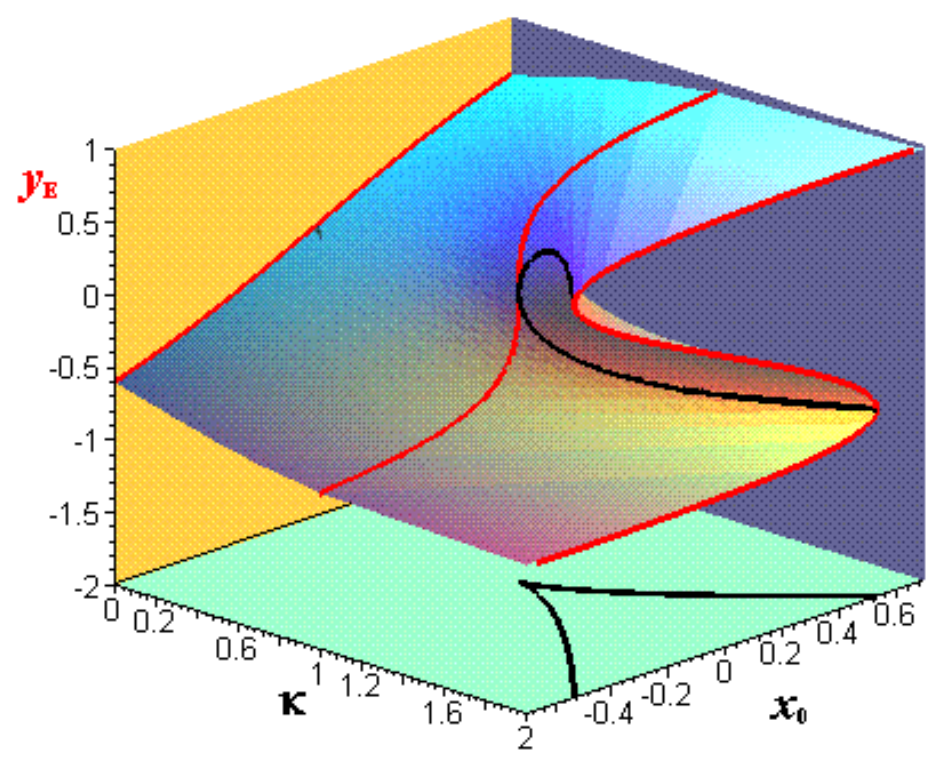

Figure 4.1: The surface of extrema $y_{E}\left(x_{0} ; \kappa, \gamma=1\right)$ of the potential $V\left(y ; x_{0}, \kappa, \gamma=\right.$ $1, \nu=1)$ over the $\left(x_{0} ; \kappa\right)$ plane with the cusp-curve separating threefold and unique domains

\subsection{Political Opinion Formation in Terms of Catastrophe Theory}

We now focus on the limit case (4.4) of a very steadfast (i. e. slowly changing) inner inclination $x(\tau) \approx x_{0}$. The motion of $y(\tau)$ then follows in a very good approximation the gradient of $V\left(y, x_{0} ; \kappa, \gamma, \mu=1-\varepsilon\right)$ at the momentary value $x_{0}$ of the slow variable. Then, and only then, it can be described in the stylized and illustrative terms of catastrophe theory. We discuss two important cases.

- The first case is a liberal society with very low opinion pressure $\kappa \ll 1$ belonging to domain $\mathbb{D}_{0}$ in the $\left(x_{0}, \kappa\right)$ plane. The potential $V\left(y, x_{0} ; \kappa\right)$ has exactly one minimum and the surface $\partial V / \partial y=0$ is unique. According to equation (4.5) the variable $y(\tau)$ will relax along the slope of the potential towards its "equilibrium value" $y_{E}=\tanh \left(\kappa y_{E}+\gamma x_{0}\right)$ determined uniquely by $x_{0}, \kappa$ and $\gamma$. If $x_{0}$ moves slowly $y(\tau)$ will then follow. It is consistent with this dynamics to consider $x$ (the inner inclination) as causative variable and $y$ (the majority variable) as reactive variable. This assumes prevailingly a bottom-up interaction between the individual and collective level.

- The second case assumes an extremely totalitarian society with high opinion pressure $(\kappa=2.0)$ and simultaneously a high disapproval trend $(\beta=-1.2)$. Suppose the inner inclination $x_{0}$ is very steadfast (i. e. developing very slowly but consistently with $\mu=0.02 \ll 1$ ). This case (see scenario 8) can be treated in terms of catastrophe theory, making use of the potential $V\left(y, x_{0} ; \kappa=2.0, \gamma=1, \beta=-1.2\right)$. In the totalitarian domain $(\kappa>1)$ the potential has for $|x|<\left|x_{c}\right|$ two minima and one maximum in between and for $|x|>\left|x_{c}\right|$ one minimum only. The corresponding holds for the surface $\partial V / \partial y=0$ with the unique and threefold part (see figure 4.1). 


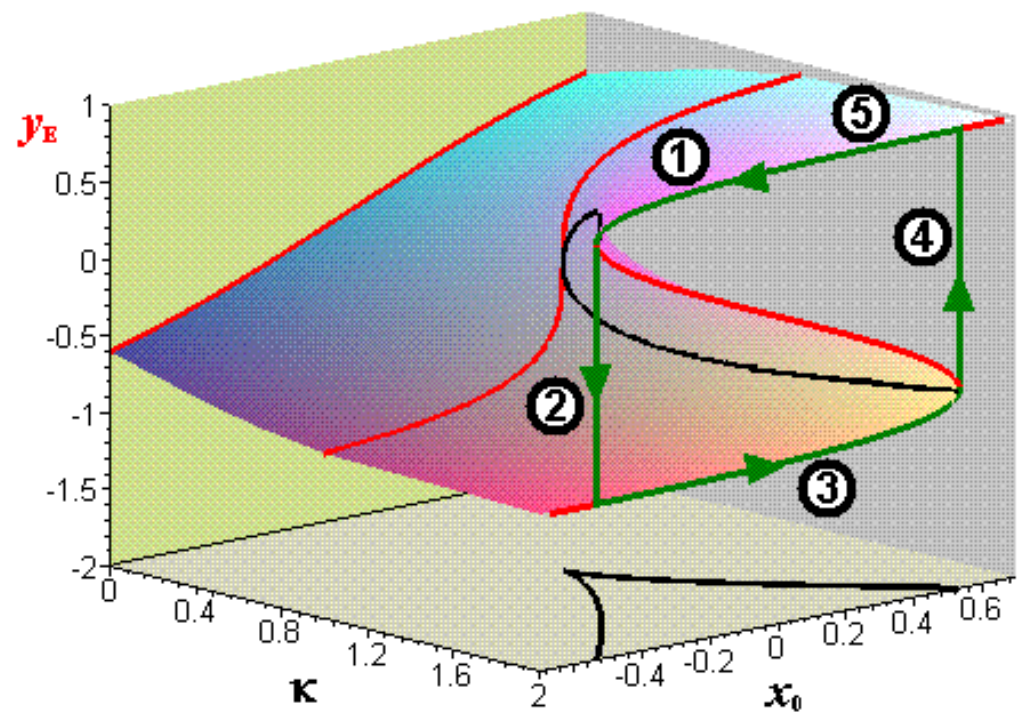

Figure 4.2: The hysteresis loop traversed by a totalitarian society with $\kappa=2.0$ in the $\left(y_{E}, x_{0}, \kappa\right)$ space

The society of scenario 8 traverses in a very good approximation the "hysteresis loop" shown in figure 4.2. This represents in the stylized version of catastrophe theory the limit cycle in scenario 8.

We now distinguish five stages of dynamics and the pertaining shape of the potential engendering this dynamics (see figure 4.3).

- Initial state:

We start with an indifferent inclination $x_{0}=0$ whereas $y(\tau)$ has already reached the equilibrium value $y_{E+}\left(x_{0}=0\right)$, one of the potential minima.

- Stage 1

The disapproval trend $\beta=-1.2$ has slowly drawn the inner inclination to $x_{0}=-x_{c}$ and changed the potential. $y(\tau)$ has slowly reached $y_{E+}\left(x_{0}=-x_{c}\right)$.

- Stage 2

The potential minimum at $y_{E+}\left(x_{0}=-x_{c}\right)$ disappears. While $x_{0}(\tau)$ stays at $-x_{c}$ the majority variable $y(\tau)$ jumps quickly from $y_{E+}\left(-x_{c}\right)$ to $y_{E-}\left(-x_{c}\right)$, the only surviving minimum.

- Stage 3

Drawn by the disapproval trend $x_{0}(\tau)$ moves slowly from $-x_{c}$ to $+x_{c}$. Simultaneously $y(\tau)$ slowly traverses the equilibrium values from $y_{E-}\left(-x_{c}\right)$ via $y_{E-}\left(x_{0}=0\right)$ to $y_{E-}\left(x_{c}\right)$. The potential changes correspondingly.

- Stage 4

The potential minimum at $y_{E-}\left(x_{c}\right)$ disappears. While $x_{0}(\tau)$ stays at $+x_{c}$ the majority $y(\tau)$ jumps quickly from $y_{E-}\left(x_{c}\right)$ to $y_{E+}\left(x_{c}\right)$.

- Stage 5

Drawn by the disapproval trend $x_{0}(\tau)$ slowly moves from $x_{c}$ to $x_{0}=0 . y(\tau)$ slowly traverses the equilibrium values from $y_{E+}\left(x_{c}\right)$ to $y_{E+}\left(x_{0}=0\right)$. The system has returned to the initial state.

Evidently there occurs a dramatic change between 


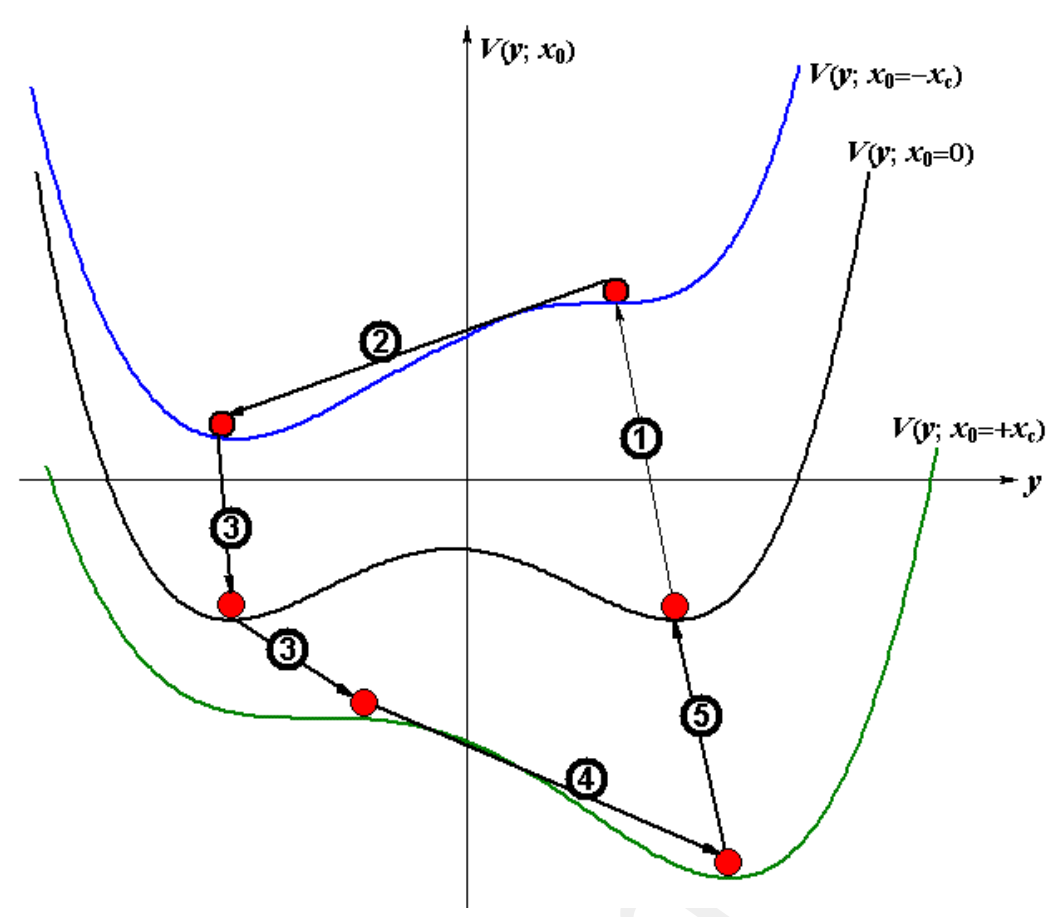

Figure 4.3: Stages of dynamics traversed by a totalitarian society and pertaining shape of the potential

- stages 1, 3 and 5 where $y(\tau)$ stays in quasi-equilibrium and

- the revolutionary stages 2 and 4 where $y(\tau)$ loses one collectively stabilized equilibrium and jumps quickly into the equilibrium belonging to the "antiideology".

The mathematical reason for the differences between the slow quasi-equilibrium stages and the fast revolutionary stages is apparent:

- In the stagnant stages 1,3 and 5 the motion of $y(\tau)$ and $x(\tau)$ is slow because $y(\tau)$ is in the vicinity of the equilibrium value $y_{E+}$ respectively $y_{E-}$ and $x_{0}(\tau)$ moves slowly with $\mu \ll 1$.

- In the revolutionary stages 2 and 4 the motion of $y(\tau)$ is fast because $y(\tau)$ must run down the slope of $V\left(y, x_{0}, \kappa, \gamma, \nu\right)$ in order to reach the only remaining minimum of $V$. The motion of $x_{0}(\tau)$ remains slow due to $\mu \ll 1$.

\section{Acknowledgement}

One of us (W.W.) is very grateful for intense discussions with and highly valuable advice from Dr. Igor Chernenko. He is an expert on catastrophe theory and its applications (see for instance Chernenko 1999) and has drawn our attention to relating the model with the concepts of catastrophe theory and helped us in representing these concepts appropriately. Furthermore W.W. wants to thank professor Gerrit Broekstra for very stimulating discussions about his and his coauthors' work (Broekstra et al. 2005). By appropriate re-definition he has applied 
the model to the important and completely different case of the formation of buy or sell decisions among investors.

\section{References}

Bigelow, J., 1982. A catastrophe model of organisational change. Behavioral Science 27, 26-42.

Broekstra, G., Sornette, D., Zhou, W.X., 2005. Bubble, critical zone and crash of Royal Ahold.

Physica A, 346, 529-560.

Chernenko, J.V., 1999. Cargo cult science and the catastrophe theory

Nonlinear Analysis 38, 87-103.

Farraro, T. J., 1978. An introduction to catastrophes. Behavioral Science 23, 291-318.

Flay, B.R., 1978. Catastrophe theory applications in psychology. Behavioral Science 23, 335-350.

Guastello, S.J., 1988. The organisational security subsystem: some potentially catastrophic events.

Behavioral Science 33, 48-57.

Guastello, S.J., 1995 Chaos, catastrophe, and human affairs. Mahwah, NJ: Lawrence Erlbaum Associates.

Haken, H., 1977. Synergetics - an introduction. Nonequilibrium phase transitions and self-organisation in physics, chemistry and biology. Berlin, Heidelberg, New York: Springer.

Klemperer, V., 1999. I will bear witness: a diary of the Nazi years 19331941.

New York: Modern Library;

Original title: Ich will Zeugnis ablegen bis zum letzten.

Berlin: Aufbau Taschenbücher (1999).

Leonhard, W., 1979. Child of the revolution.

London: Pluto Press.

Original title: Die Revolution entlässt ihre Kinder.

Köln (1955): Kiepenheuer \& Witsch.

Poston, T., Stewart, I., 1978. Catastrophe theory and its application. London: Pitman.

Rosser, J.B. Jr., 2000. From catastrophe to chaos: A general theory of economic discontinuity, $2^{\text {nd }}$ ed., Vol. 1: Mathematics, Microeconomics, Macroeconomics, and Finance.

Boston: Kluwer.

Thom, R., 1972. Stabilité structurelle et morphogénèse.

New York: Benjamin.

Weidlich, W., 1971. The statistical description of polarisation phenomena in society.

British Journal of Mathematical and Statistical Psychology 24, 251266. 
Weidlich, W., 1991. Physics and social science - the approach of synergetics. Physics Reports Vol. 204(1), 1-163.

Weidlich, W., 1994. Synergetic modelling concepts for sociodynamics with application to collective political opinion formation.

Journal of Mathematical Sociology 18, 267-291.

Weidlich, W., 2000. Sociodynamics - a systematic approach to mathematical modelling in the social sciences.

Amsterdam: Gordon and Breach (2000).

London: Taylor and Francis (2002).

Moskow: YPCC (2004).

Mineola, New York: Dover Publications (2006).

Tokyo: Morikita Shuppan (2007).

Weidlich, W., 2005. Thirty years of sociodynamics. An integrated strategy of modelling in social sciences: application to migration and urban evolution.

Chaos, Solitons and Fractals 24, 45-56.

Weidlich, W., 2006. Intentions and principles of sociodynamics.

Evol. Inst. Econ. Rev. 2 (2), 161-165.

Weidlich, W., Haag, G. 1983. Concepts and models of a quantitative sociology.

Berlin, Heidelberg, New York: Springer.

Zeeman, E.C., 1977. Catastrophe theory.

München, Amsterdam: Addison-Wesley. 